February 2016

"Simple Centrifugal Incentives in Spatial Competition "

Didier Laussel, Michel Le Breton, Dimitrios Xefteris 


\title{
Simple Centrifugal Incentives in Spatial Competition*
}

\author{
Didier Laussel $^{\dagger} \quad$ Michel Le Breton ${ }^{\ddagger} \quad$ Dimitrios Xefteris $^{\S}$
}

February 2016

\begin{abstract}
This paper studies the effects of introducing centrifugal incentives in an otherwise standard Downsian model of electoral competition. First, we demonstrate that a symmetric equilibrium is guaranteed to exist when centrifugal incentives are induced by any kind of partial voter participation (such as abstention due to indifference, abstention due to alienation, etc.) and, then, we argue that: a) this symmetric equilibrium is in pure strategies, and it is hence convergent, only when centrifugal incentives are sufficiently weak on both sides; b) when centrifugal incentives are strong on both sides (when, for example, a lot of voters abstain when they are sufficiently indifferent between the two candidates) players use mixed strategies - the stronger the centrifugal incentives, the larger the probability weight that players assign to locations near the extremes; and c) when centrifugal incentives are strong on one side only - say for example only on the right - the support of players' mixed strategies contain all policies except from those that are sufficiently close to the left extreme.
\end{abstract}

JEL classification codes: D71, D72.

Keywords: Electoral Competition; Spatial Model; Downs; Mixed equilibria; Centrifugal incentives; Abstention.

\section{Introduction}

In a standard Downsian model of electoral competition, ${ }^{1}$ a candidate located, for example, to the left of her competitor always has incentives to approach her competitor's location because all voters with ideal

${ }^{*}$ The authors would like to thank two referees, an associate editor, Philippe De Donder and Juuso Välimäki as well as participants of CRETE2013, EPSA General Conference 2014 and the MFS 2015 Conference for excellent comments and suggestions.

${ }^{\dagger}$ Aix-Marseille University (Aix Marseille School of Economics, CNRS and EHESS).

${ }^{\ddagger}$ Institut Universitaire de France and Toulouse School of Economics (University of Toulouse Capitole).

$\S$ University of Cyprus.

${ }^{1}$ We refer to Duggan (2012) and Osborne (1995) for nice surveys of the literature which originates to Hotelling's (1929) and Downs's (1957) models of spatial competition. 
policies to her left will still vote for her. That is, a player in such a model unambiguously increases her vote-share by approaching her competitor. In a sense, the standard Downsian model takes in account existence only of centripetal incentives (understood here broadly as incentives that make one player want to move closer to her opponent) and hence the popular convergence-to-the-center result is obtained.

But what if a share of voters located to the left (right) of the most leftist (rightist) candidate (or party) feels alienated from the political process, and prefers to abstain or vote for extremist candidates instead of the two main ones? What if a share of voters located in between candidates feels indifferent and prefers to abstain from voting? In such cases it is straightforward that any motion of a candidate towards her opponent is not unambiguously profitable - such voters' behaviors generate centrifugal incentives that mitigate the centripetal ones. To quote Cox (1990), from whom we borrow this terminology, centrifugal incentives should be expected to "lead to the advocacy of, more or less, extreme positions". ${ }^{2}$ Hence, introduction of centrifugal incentives in an otherwise standard spatial model should be expected to destabilize the convergence-to-the-center result.

In this paper we consider a generalized Downsian model that takes in account such centrifugal incentives. We assume that a participating voter ${ }^{3}$ always behaves in the standard manner: she votes for the candidate whose policy proposal is closer to her ideal policy. But the share of participating voters of a certain preference type (that is, of voters that have the same policy preferences), is allowed to be essentially any function of candidates' policy proposals - which may different, of course, for every distinct voters' type. That is, our model takes in account all kinds of cases of partial voter participation (for example, abstention due to indifference, ${ }^{4}$ abstention due to alienation, ${ }^{5}$ etc.) and this makes us believe that its analysis is relevant and worthwhile.

We notice that this general formulation is equivalent to having that the share of participating voters to the left (right) of the most leftist (rightist) candidate and the share of participating voters in between the platform of the most leftist (rightist) candidate and the ideal policy of the indifferent voter, are functions

\footnotetext{
${ }^{2}$ For a detailed exposition of possible reasons behind centrifugal incentives in electoral competition one is referred to Adams, Merrill and Grofman (2005). We briefly discuss some of them in the end of this section.

${ }^{3} \mathrm{~A}$ participating voter is someone who votes for one of the two main competing candidates. On the contrary a nonparticipating voter is a voter that does not vote for one of these two candidates: such voters could be abstaining or they could be voting for other non-instrumental candidates. In the Appendix we provide a specific application in which nonparticipating voters are assumed to vote for extremist/niche parties with fixed policy positions (non-instrumental).

${ }^{4}$ For example, Matakos et al. (2015b) introduce indifference-based abstention to a unidimensional model of electoral competition in order to study the effect of electoral rule disproportionality on turnout (one is referred to Matakos et al., 2015a, for a more comprehensive presentation of the formal setup) and show that it generates centrifugal incentives.

${ }^{5}$ Downs (1957) shows that alienation-based abstention reinforces centrifugal incentives (conditional on the distribution of voters' ideal policies not being very polarized).
} 
of the policy platforms of the two candidates. Indeed, candidates' location choices (when they are interior and they do not coincide) divide the space in four regions ${ }^{6}$ - two peripheral regions and two central ones - and hence, for a given distribution of ideal policies, we essentially have four (possibly distinct) functions that determine the share of voters that participate in the election - one function for each region.

The first contribution of the paper is to prove that for this general class of centrifugal incentives (when, centrifugal incentives arise due to partial voter participation), the game admits a symmetric Nash equilibrium for every possible distribution of voters' ideal policies. Since this generalized model is a discontinuous one (like the standard Downsian one), in order to establish this general existence result we apply a theorem of Dasgupta and Maskin (1986). We then try to shed some light on the nature of the symmetric equilibria that we proved to exist by focusing on the simplest class of centrifugal incentives. That is, by focusing on the simplest kind of functions that could be used to determine the share of participating voters in each region - the class of constant functions - and on a uniform distribution of voters' ideal policies. Notice that the use of constant and generally different alienation functions introduces in our model a second ingredient besides centrifugal incentives: differential party loyalty. Namely, when the share of voters located to the left of the most leftist candidate who feel alienated from the political process differs from the share of voters located to the right of the most rightist candidate who feel alienated from the political process, there is generally a discontinuity of a party's payoff function when its rival is located at the center. This means that being identified as the leftist (or the rightist) party, by differentiating one's policy proposals even infinitesimally from the rival party's policy proposals, induces an upward (downward) jump in a party's vote share.

In general three types of configurations may be encountered.

The first one describes the case where centripetal incentives dominate centrifugal incentives on both sides of the strategy space (large shares of participating voters in the peripheral regions compared to the central ones). In that case, the Downsian logic of minimal differentiation, where the two players converge towards a point which may be more or less close to the center, depending on the degree of asymmetry between the centrifugal incentives, applies. For the uniform distribution, the point of convergence will belong to the interval $\left[\frac{1}{3}, \frac{2}{3}\right]$. This is because even if centripetal incentives are dominant on both sides they can still be asymmetric; asymmetry of these forces crucially affects the point of convergence. If, for

\footnotetext{
${ }^{6}$ The first region contains all locations to the left of the most leftist platform, the second region contains all locations from the most leftist platform to the ideal policy of the indifferent voter (i.e. the voter whose ideal policy is equidistant from the two platforms), the third region contains all locations in between the ideal policy of the indifferent voter and the most rightist platform and the fourth region all locations to the right of the most rightist platform.
} 
example, players face stronger centrifugal incentives from the right (the share of participating voters of the left peripheral region is larger than that of the right one), they converge to a point which belongs on the left half of the strategy space. That is, on the side of the policy space where centrifugal incentives are weaker - not the other way round. This is intuitively the consequence of the differential party loyalty effect. In the previous example, when the rival party located at the center, a party would unambiguously increase its vote-share by choosing a policy proposal just to the left of its rival's, allowing to be identified itself as the leftist party. ${ }^{7}$ Most importantly we show that candidates' choices in this case depend only on the shares of participating voters in the peripheral regions.

The second configuration describes the case where centrifugal incentives dominate centripetal incentives on both sides of the strategy space (small shares of participating voters in the peripheral regions compared to the central ones). This case raises some interesting coordination problems if the players are totally opportunistic, that is, without real attachment to the left or the right. If a coordination mechanism is absent, there is a symmetric equilibrium in mixed strategies. Not surprisingly, the expected degree of differentiation in this equilibrium is increasing in the degree of the centrifugal incentives. In the case in which centrifugal incentives on both sides are of equal magnitude, there is a smooth transition from these mixed equilibria to the pure strategy equilibrium of the strong centripetal incentives case. When centrifugal incentives are very large, players use a dispersed mixed strategy which assigns large probability weights to locations at the extreme of the strategy space (U-shaped density function). As the magnitude of centrifugal incentives declines, but as long as they dominate centripetal ones, the mixed strategy that players use assigns an increasing probability weight to central locations (inverse U-shaped density). In this case, candidates' expected location depends only on the shares of participating voters in the central regions.

The third configuration describes a situation where centripetal incentives are dominant on one side and centrifugal incentives are dominant on the other side. ${ }^{8}$ Assume, for example, that centripetal forces dominate on the left and centrifugal forces dominate on the right (large share of participating voters on the left and small share of participating voters on the right). This configuration is non-trivial as there is no equilibrium in pure strategies: the best reply dynamics are chaotic. We characterize a symmetric equilibrium in mixed strategies which has a number of interesting features. The common mixed strategy that players use in this equilibrium is such that a) the support of this mixed strategy does not contain

\footnotetext{
${ }^{7}$ Remember that, in this example, centripetal incentives (and, hence, party loyalty) are stronger on the left.

${ }^{8}$ There is some similarity between this configuration and the unidirectional Hotelling-Downs 's model analyzed in Cancian, Bergström and Bills (1995), Gabszewicz, Laussel and Le Breton (2008) and Xefteris (2013).
} 
extreme leftist locations, b) the support of this mixed strategy contains extreme rightist locations, c) the density of the underlying distribution of this mixed strategy is decreasing and d) candidates' expected location depends on the shares of participating voters in both central and peripheral regions.

Of course, we are not the first ones to consider unidimensional models of electoral competition with both centripetal and centrifugal incentives. The post-Downsian literature contains several models along these lines. In particular, in the framework of electoral competition centrifugal incentives have been introduced through a variety of different channels. For instance in a model with ideological candidates and uncertainty on the location of the median voter (Roemer, 2001), the leftist candidate will balance between moving left to please (if elected) the voter on behalf of whom he is acting and moving towards the centre to increase the probability of winning the election. Moreover, Adams, Merrill and Grofman $(2005)^{9}$ "show that three factors, each linked to voter choice, can generate strong centrifugal pressures on the positioning of parties: (1) the existence of non-policy considerations in voter decision making most notably party loyalty; (2) the capacity of voters to discount the claims of candidates concerning the policy changes they could achieve; and (3) an unwillingness of citizens to participate in the political process when they find that none of the existing parties or candidates are sufficiently attractive". ${ }^{10}$ While the authors recognize that such games generally fail to admit a Nash equilibrium in pure strategies, they do not investigate the corresponding class of patterns to which they refer as unstable. ${ }^{11}$ Instead, they determine conditions under which the game admits a unique Nash equilibrium in pure strategies. In this paper we characterize a symmetric equilibrium in mixed strategies for a simple class of centrifugal incentives, which allows us, though, to study in detail the effect of a wide range of asymmetries among these incentives on players' equilibrium behavior.

In what follows we describe the model (section 2), we present some general results, mainly regarding equilibrium existence (section 3), we conduct a detailed equilibrium analysis for the case in which centrifugal incentives are modelled by a quadruple of constant functions and the voters' ideal policies are uniformly distributed on the policy space (section 4) and, finally, we conclude (section 5). In the Appendix we provide some results about pure strategy equilibria for nonuniform distributions of voters'

\footnotetext{
${ }^{9}$ See also Merrill and Adams (2002) who analyze factors that affect candidates' position-taking incentives in multicandidate and multi-party elections. For a multivariate vote model that includes a Left-Right policy component, a party identification component and an unmeasured term that renders the vote choice probabilistic, they present theoretical and computer simulation results that quantify candidates' incentives to shift their policies away from the center in the direction of their partisan constituencies' mean policy preferences. Centrifugal incentives are found to increase with (1) the salience of policies and party identification, (2) the size of the candidate field, (3) the size of a candidate's partisan constituency and (4) more extreme constituency policy preferences. Thus, ceteris paribus, candidates who represent large constituencies are motivated to present more extreme policies than are candidates who represent small ones.

${ }^{10}$ Page 2.

${ }^{11}$ Page 44.
} 
ideal policies and also some microfoundations of the constant functions extensively analyzed in the paper.

\section{The Model}

We consider a model in which two players (candidates/parties), 1 and 2, simultaneously locate on the interval $[0,1]$. We denote by $x(y)$ the location choice of player $1(2)$. The ideal policies of a unit mass of voters are distributed on $[0,1]$ according to a strictly increasing and absolutely continuous distribution function, $F:[0,1] \rightarrow[0,1]$. Given a profile $(x, y) \in[0,1]^{2}$, the payoffs of the two players are assumed to be as follows

$$
\pi_{1}(x, y)=\left\{\begin{array}{l}
\int_{0}^{\frac{x+y}{2}} \Xi(\theta, x, y) f(\theta) d \theta \text { if } x<y \\
\frac{1}{2} \int_{0}^{1} \Xi(\theta, x, y) f(\theta) d \theta \text { if } x=y \\
\int_{\frac{x+y}{2}}^{1} \Xi(\theta, x, y) f(\theta) d \theta \text { if } x>y
\end{array}\right.
$$

and the payoff of candidate 2 is given by:

$$
\pi_{2}(x, y)=\left\{\begin{array}{l}
\int_{0}^{\frac{x+y}{2}} \Xi(\theta, x, y) f(\theta) d \theta \text { if } y<x \\
\frac{1}{2} \int_{0}^{1} \Xi(\theta, x, y) f(\theta) d \theta \text { if } y=x \\
\int_{\frac{x+y}{2}}^{1} \Xi(\theta, x, y) f(\theta) d \theta \text { if } y>x
\end{array}\right.
$$

where $\Xi$ is integrable with respect to $\theta$ on $[0,1]$, takes non-negative values at most as large as one and is symmetric - that is, $\Xi(\theta, x, y)=\Xi(\theta, y, x)$ for every $(x, y) \in[0,1]^{2}$. The postulate behind these payoff functions is that, given a menu of two platforms $x$ and $y$, a participating voter always votes for the candidate who proposes the policy nearer to her ideal policy, but only a fraction, $\Xi(\theta, x, y)$, of voters with ideal policy $\theta$ actually participate in the election. Since $\Xi(\theta, x, y)$ is allowed to vary in all its arguments the above formulation captures essentially all kinds of centrifugal incentives that may arise due to partial voter participation. In studies which consider abstention due to indifference (see, for example, HortalaVallve and Esteve-Volart 2011 and Matakos et al. 2015b), $\Xi(\theta, x, y)$ is zero (one) for every $\theta$ sufficiently close to (far from) $\frac{x+y}{2}$ while in studies which consider abstention due to alienation (see, for example, Downs 1957), $\Xi(\theta, x, y)$ is zero (one) for every $\theta$ sufficiently far from (close to) candidates' platforms.

For a given distribution $F$, we can define $\alpha(x, y)=\frac{\int_{0}^{\min \{x, y\}} \Xi(\theta, x, y) f(\theta) d \theta}{F(\min \{x, y\})}$ for $\min \{x, y\}>0$ and 
$\alpha(x, y)=\alpha \in[0,1]$ otherwise; $\beta(x, y)=\frac{\int_{\max \{x, y\}}^{1} \Xi(\theta, x, y) f(\theta) d \theta}{1-F(\max \{x, y\})}$ for $\max \{x, y\}<1$ and $\beta(x, y)=\beta \in[0,1]$ otherwise; $\gamma(x, y)=\frac{\int_{\min \{x, y\}}^{\frac{x+y}{2}} \Xi(\theta, x, y) f(\theta) d \theta}{F\left(\frac{x+y}{2}\right)-F(\min \{x, y\})}$ for $x \neq y$ and $\gamma(x, y)=\gamma \in[0,1]$ otherwise; and $\delta(x, y)=$

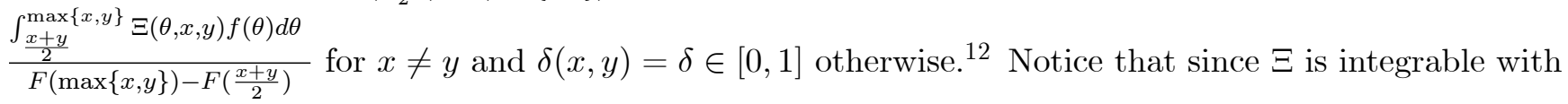
respect to $\theta$, all the above functions are guaranteed to be continuous everywhere, except possibly at their problematic points.

Hence we can re-write the players' payoff functions as:

$$
\pi_{1}(x, y)= \begin{cases}\alpha(x, y) F(x)+\gamma(x, y)\left(F\left(\frac{x+y}{2}\right)-F(x)\right) & \text { if } x<y \\ \frac{1}{2}[\beta(x, y)(1-F(x))+\alpha(x, y) F(x)] & \text { if } x=y \\ \beta(x, y)(1-F(x))+\delta(x, y)\left(F(x)-F\left(\frac{x+y}{2}\right)\right) & \text { if } x>y\end{cases}
$$

and

$$
\pi_{2}(x, y)= \begin{cases}\beta(x, y)(1-F(y))+\delta(x, y)\left(F(y)-F\left(\frac{x+y}{2}\right)\right) & \text { if } x<y \\ \frac{1}{2}[\beta(x, y)(1-F(x))+\alpha(x, y) F(x)] & \text { if } x=y \\ \alpha(x, y) F(y)+\gamma(x, y)\left(F\left(\frac{x+y}{2}\right)-F(y)\right) & \text { if } x>y\end{cases}
$$

Given a) that this model incorporates a wide class of diverse cases of centrifugal incentives and b) that this second pair of payoff functions offers an arguably more direct way of classifying these cases into subgroups (based on how voters behave on average in each of the four distinct regions), we stick with the latter formulation throughout our analysis.

Before we proceed though we draw attention to some features of the game that need to be emphasized. First, notice that for a generic quadruplet $(\alpha, \beta, \gamma, \delta)$, the game is not zero-sum. In spite of the fact that the game is competitive, it also contains coordination dimensions. Second, the game is symmetric in the sense that $\pi_{1}(x, y)=\pi_{2}(y, x)$ for all $(x, y) \in[0,1]^{2}$. Third, the game is discontinuous: the function is discontinuous on the diagonal of the square. Symmetric discontinuous games raise intricate difficulties as they do not necessarily admit equilibria in mixed strategies and, even if they do, they do not always admit a symmetric equilibrium (see Fey 2012 and Xefteris 2015). In this paper, we prove existence of a symmetric equilibrium for the general case using the Dasgupta and Maskin (1986) conditions and then we

\footnotetext{
${ }^{12}$ Setting the functions equal to some arbitrary constant at their problematic points is only made for completeness and has no effect on formal analysis.
} 
construct explicitly a symmetric equilibrium for the case in which $(\alpha, \beta, \gamma, \delta)$ is a quadruplet of constant functions and $F$ is uniform.

\section{General Remarks}

The model that we consider introduces, principally, centrifugal forces and, secondarily, differential party loyalty. On one hand, when the centrifugal (centripetal) incentives are dominant on the left (right), the vote-share of a party decreases (increases) when a party approaches her opponent from the left (right). On the other hand, differential party loyalty results generally in a discontinuity of the party's payoff function when the party's policy proposition becomes, at the center, identical to its rival's one. As already argued, this reflects the possibly different magnitudes of the centrifugal incentives on the left of the leftist party and on the right of the rightist one.

For the sake of illustration, consider that $\alpha(x, y)=\alpha, \beta(x, y)=\beta, \gamma(x, y)=\gamma>0$ and $\delta(x, y)=\delta>0$ for every $(x, y) \in[0,1]^{2}$; and define $\hat{\alpha}=\alpha / \gamma$ and $\hat{\beta}=\beta / \delta$. Then, if the second player locates to the right of the first player $(x<y)$, we have:

$$
\frac{\partial \pi_{2}}{\partial y}(x, y)=\left[(1-\hat{\beta}) f(y)-\frac{1}{2} f\left(\frac{x+y}{2}\right)\right] \delta
$$

and therefore:

$$
\frac{\partial \pi_{2}}{\partial y}(x, y)<0 \text { if and only if }(1-\hat{\beta}) f(y)-\frac{1}{2} f\left(\frac{x+y}{2}\right)<0 .
$$

If $\hat{\beta}$ is close to one, not surprisingly, the inequality is likely to hold true as the right player does not lose too much voters on its right by moving to the left. On the contrary, when $\hat{\beta}=0$, the condition writes $f(y)<\frac{1}{2} f\left(\frac{x+y}{2}\right)$. If the density does not decrease too fast, it will not be satisfied. For instance, when $F$ is uniform, it does not hold true. Precisely, when $F$ is uniform, the general inequality holds true if and only if $\hat{\beta}>\frac{1}{2}$. For this reason when we refer to strong centrifugal (centripetal) incentives we actually mean small (large) values of $\hat{\alpha}$ and $\hat{\beta}$.

The conventional Hotelling-Downs model corresponds to $\hat{\alpha}=\hat{\beta}=1$ and, hence, it pays attention exclusively to centripetal forces, that is, incentives pushing each player to move in the direction of its 
opponent. Centrifugal incentives are absent. On the contrary, our model takes into account a variety of centrifugal forces. To understand the nature of these implications, we look at the payoff function of the first player when the distribution of voters is uniform and $\gamma=\delta$.

Let $\hat{\alpha}, \hat{\beta}>\frac{1}{2}$. In such a case the centripetal incentives are strong and, thus, the payoff function $\pi_{1}$ is increasing on $[0, y)$, decreasing on $(y, 1]$ and displays a discontinuity at $y$ if and only if $y \neq \frac{\beta}{\alpha+\beta}$. The discontinuity is as depicted in figure $1 a$ if $y>\frac{\beta}{\alpha+\beta}$ or as depicted in figure $1 b$ if $y<\frac{\beta}{\alpha+\beta}$.

Insert Figure 1 here

Now, let $\hat{\alpha}, \hat{\beta}<\frac{1}{2}$. In such a case, the centrifugal incentives are strong and, thus, the payoff function $\pi_{1}$ is decreasing on $[0, y)$, increasing on $(y, 1]$ and displays a discontinuity at $y$ if and only if $y \neq \frac{\beta}{\alpha+\beta}$. Since $\pi_{1}(0, y)=\gamma \frac{y}{2}, \pi_{1}(1, y)=\delta \frac{1-y}{2}=\gamma \frac{1-y}{2}$ and $\pi_{1}(y, y)=\gamma \frac{\hat{\alpha} y+\hat{\beta}(1-y)}{2}$, the best response of player 1 to $y$ is zero if $y>\frac{1}{2}$ and one if $y<\frac{1}{2}$. Indeed, since $\hat{\alpha}<\frac{1}{2}$, we cannot have both $\frac{y}{2}<\frac{\hat{\alpha} y+\hat{\beta}(1-y)}{2}$ and $\frac{1-y}{2}<\frac{\hat{\alpha} y+\hat{\beta}(1-y)}{2}$. But the value at one extreme may be smaller than the value at the discontinuity point. The graph of $\pi_{1}$ is as depicted in figure $1 c$ if $y>\frac{\beta}{\alpha+\beta}$ or as depicted in figure $1 d$ if $y<\frac{\beta}{\alpha+\beta}$.

Finally, let $\hat{\alpha}>\frac{1}{2}>\hat{\beta}$. In such case, the centrifugal incentives are dominant on the right and the centripetal incentives are dominant on the left and, thus, the payoff function $\pi_{1}$ is increasing on $[0, y)$, increasing on $(y, 1]$ and displays a discontinuity at $y$ if and only if $y \neq \frac{\beta}{\alpha+\beta}$. The graph of $\pi_{1}$ is as depicted in figure $1 e$ if $y>\frac{\beta}{\alpha+\beta}$ or as depicted in figure $1 f$ if $y<\frac{\beta}{\alpha+\beta}$.

Equilibrium existence in such discontinuous games is not straightforward. Our first task will be to demonstrate that our game satisfies the Dasgupta and Maskin (1986) conditions which guarantee the existence of a symmetric equilibrium in mixed strategies ${ }^{13}$ in certain symmetric games with discontinuities.

Proposition 1 The game admits a symmetric Nash equilibrium in mixed strategies for every admissible quadruplet of functions $(\alpha, \beta, \gamma, \delta)$ and every absolutely continuous $F$.

\footnotetext{
${ }^{13}$ For a symmetric mixed Nash equilibrium to be meaningful in a political competition setting, we must have in mind situations when there is no cost for an actor to be ultimately on the left or on the right of any other actor. There should be no constraints on positioning and leapfrogging should not be costly. This may happen if the parties are not too much ideological (like Aragonès and Palfrey, 2002, and Aragonès and Xefteris, 2013, who characterize mixed equilibria of the Downsian model with a favored candidate under perfect and imperfect information) or within a party if the competition describes primaries among candidates.
} 
Proof. Dasgupta and Maskin (1986) show that if a) the pure strategy space for each player is represented by a closed interval, $b$ ) the payoff functions are continuous except on a set of measure zero, c) the players' cumulative payoff function is upper semi-continuous, $d$ ) the range of the payoff function of each player is bounded and e) the payoff function of each player is weakly lower semi-continuous for any given strategy of the other player, then the game admits an equilibrium in mixed strategies. Therefore, to prove that our game admits a Nash equilibrium in mixed strategies the only thing that we have to do is to show that all these five conditions are met.

a) The pure strategy space for each player is $[0,1]$; a closed interval.

b) $\pi_{1}(x, y)$ and $\pi_{2}(x, y)$ are continuous except for the main diagonal, that is, except for $x=y$. This line obviously represents a measure zero of all the possible pure strategy profiles which are given by $[0,1]^{2}$.

c) $\pi_{1}(x, y)+\pi_{2}(x, y)=\alpha(x, y) F(\min \{x, y\})+\gamma(x, y)\left[F\left(\frac{x+y}{2}\right)-F(\min \{x, y\})\right]+\delta(x, y)[F(\max \{x, y\}-$ $\left.\left.F\left(\frac{x+y}{2}\right)\right)\right]+\beta(x, y)(1-F(\max \{x, y\}))$ is a continuous function since it is a sum of continuous functions. ${ }^{14}$ That is, it is upper semi-continuous as well.

d) $0 \leq \pi_{1}(x, y) \leq 1$ and $0 \leq \pi_{2}(x, y) \leq 1$ for any $(x, y) \in[0,1]^{2}$. That is, the players' payoffs are bounded.

e) $\pi_{1}(x, y)$ is weakly lower semi-continuous in $x$ if $\forall x \in[0,1], \exists \lambda \in[0,1]$ such that for $y=x$,

$$
\lambda \lim \inf _{x \rightarrow-y} \pi_{1}(x, y)+(1-\lambda) \lim \inf _{x \rightarrow+y} \pi_{1}(x, y) \geq \pi_{1}(x, y)
$$

If $y \in(0,1)$ then observe that for $y=x, \pi_{1}(x, y)=\frac{\alpha(y, y) F(y)+\beta(y, y)(1-F(y))}{2}, \liminf _{x \rightarrow^{-} y} \pi_{1}(x, y)=$ $\alpha(y, y) F(y)$ and $\liminf _{x \rightarrow+} \pi_{1}(x, y)=\beta(y, y)(1-F(y))$. It is evident that for $\lambda=\frac{1}{2}$ the required weak inequality becomes an equality for any quadruplet of continuous functions $(\alpha, \beta, \gamma, \delta)$ and any absolutely continuous $F$ and, thus, always holds. If $y \in\{0,1\}$ (say for example that $y=0$ ) then for $y=x, \pi_{1}(x, y)=$ $\frac{\beta(0,0)}{2}$ and $\liminf \operatorname{in}_{x \rightarrow+} \pi_{1}(x, y)=\beta(0,0)$. In this case the definition of weak lower semi-continuity requires that $\lim \inf _{x \rightarrow+} \pi_{1}(x, y) \geq \pi_{1}(x, y)$ which holds for any any quadruplet of continuous functions $(\alpha, \beta, \gamma, \delta)$

\footnotetext{
${ }^{14}$ The function $\alpha(x, y) F(\min \{x, y\})$ is continuous everywhere because when $\min \{x, y\}>0$ both $\alpha(x, y)$ and $F(\min \{x, y\})$ are continuous and when $\min \{x, y\}=0$ we have $\alpha(x, y) F(\min \{x, y\})=0$ and $\lim _{\min \{x, y\} \rightarrow 0} \alpha(x, y) F(\min \{x, y\})=0$. Equivalently, one can show that $\beta(x, y)(1-F(\max \{x, y\})), \gamma(x, y)\left[F\left(\frac{x+y}{2}\right)-F(\min \{x, y\})\right]$ and $\delta(x, y)\left[F\left(\max \{x, y\}-F\left(\frac{x+y}{2}\right)\right)\right]$ are continuous everywhere.
} 
and any absolutely continuous $F$. That is, $\pi_{1}(x, y)$ (and equivalently $\pi_{2}(x, y)$ ) is weakly lower semicontinuous and the game admits a Nash equilibrium in mixed strategies.

Finally, Dasgupta and Maskin (1986) prove (Lemma 7) that if a game that satisfies the above conditions is moreover symmetric and discontinuities are restricted on the main diagonal, ${ }^{15}$ then this game should admit a symmetric Nash equilibrium in mixed strategies. Since the game that we study is symmetric, it follows that it should admit a symmetric equilibrium.

To have a better understanding about how introduction of centrifugal incentives affects the nature of equilibria in the Downsian model, we explicitly analyze in the next section symmetric equilibria for the case in which $(\alpha, \beta, \gamma, \delta)$ is a quadruplet of constant functions and $F$ is uniform.

\section{Equilibria}

In this section, we study symmetric Nash equilibria of the game for all quadruplets $(\alpha, \beta, \gamma, \delta)$ of admissible strictly positive constant functions when $F$ is a uniform distribution on $[0,1]$. When these functions are constant we can define $\hat{\alpha}=\alpha / \gamma$ and $\hat{\beta}=\beta / \delta$. For easier presentation of the results and without loss of generality, we assume throughout this section that $\hat{\alpha} \geq \hat{\beta}$. We distinguish three broad cases according to the nature of the existing forces: centripetal $\left(\hat{\alpha}>\frac{1}{2}\right.$ and $\left.\hat{\beta} \geq \frac{1}{2}\right)$, mixed $\left(\hat{\alpha}>\frac{1}{2}>\hat{\beta}\right)$ and centrifugal $\left(\hat{\alpha} \leq \frac{1}{2}\right.$ and $\left.\hat{\beta}<\frac{1}{2}\right)$ ones. $^{16}$

Before we go to a detailed study of each case we make an observation that will prove helpful in our equilibrium exercise.

Remark 1 The discontinuity in own strategies of $\pi_{1}(x, y)$ at $y \neq \frac{\beta}{\alpha+\beta}$ and of $\pi_{2}(x, y)$ at $x \neq \frac{\beta}{\alpha+\beta}$ implies, whenever $\alpha+\beta>0$, that the two players' strategies cannot have an atom at the same point $\bar{x} \neq \frac{\beta}{\alpha+\beta}$ because each player would obtain a strictly larger payoff by choosing a platform just to the right or to the

\footnotetext{
${ }^{15}$ See the discussion in page 7 of their paper.

${ }^{16}$ For the sake of completeness we present here the equilibria of the limit case where the centrifugal and centripetal incentives balance exactly, i.e. $\hat{\alpha}=\hat{\beta}=\frac{1}{2}$. Clearly this case represents a measure zero of all possible combinations of parameters values.

If $\hat{\alpha}=\hat{\beta}=\frac{1}{2}$, then it is easy to check that there is a continuum of Nash equilibria. Precisely, up to interchangeability, $(x, y)$ with $x \leq y$ is a pure strategy Nash equilibrium if and only if $x \leq \frac{1}{2}$ and $y \geq \frac{1}{2}$. The Nash equilibria are Pareto ranked: the smaller the $x$ and the larger the $y$, the larger are the payoffs of both players.
} 
left of such an $\bar{x}$. This rules out symmetric pure strategy equilibria other than $x=y=\frac{\beta}{\alpha+\beta}$ as well as symmetric atomic mixed strategy equilibria.

Case 1 - Centripetal incentives $\left(\hat{\alpha}>\frac{1}{2}\right.$ and $\left.\hat{\beta} \geq \frac{1}{2}\right)$.

This case corresponds to a scenario in which both players increase their payoffs by approaching one another.

Proposition 2 If $\hat{\alpha}>\frac{1}{2}$ and $\hat{\beta} \geq \frac{1}{2}$ then the pure strategy profile $\left(\frac{\beta}{\alpha+\beta}, \frac{\beta}{\alpha+\beta}\right)$ is the unique pure strategy equilibrium of the game.

Proof. Consider that the first player locates strictly to the left of the second player; $x<y$. Then:

$$
\frac{\partial \pi_{1}}{\partial x}(x, y)=\gamma\left(\hat{\alpha}-\frac{1}{2}\right)>0
$$

That is, the first player has incentives to further approach the second player and hence there is no equilibrium in pure strategies such that the two players locate at distinct locations.

Now consider that both players locate at the same location; $x=y=\bar{x}$. In this case we have:

$$
\pi_{1}(\bar{x}, \bar{x})=\pi_{2}(\bar{x}, \bar{x})=\frac{1}{2}(\beta(1-\bar{x})+\alpha \bar{x})
$$

If $\bar{x}>\frac{\beta}{\alpha+\beta}$ then the first player, by deviating to $\widetilde{x}=\bar{x}-\varepsilon$, gets a payoff of:

$$
\pi_{1}(\widetilde{x}, \bar{x})=\gamma\left[\hat{\alpha}(\bar{x}-\varepsilon)+\left(\frac{2 \bar{x}-\varepsilon}{2}-(\bar{x}-\varepsilon)\right)\right]=\gamma\left(\frac{1}{2} \varepsilon+\hat{\alpha} \bar{x}-\hat{\alpha} \varepsilon\right) \rightarrow \alpha \bar{x} \text { for } \varepsilon \rightarrow 0
$$

Observe that $\bar{x}>\frac{\beta}{\alpha+\beta} \Longrightarrow \alpha \bar{x}>\beta(1-\bar{x}) \Longrightarrow \alpha \bar{x}>\frac{1}{2}(\beta(1-\bar{x})+\alpha \bar{x})$ and hence one may always find $\varepsilon>0$ small enough for such a deviation to be profitable. One can use a symmetric argument and show 
that when both players locate at $\bar{x}<\frac{\beta}{\alpha+\beta}$, the first player is better off by deviating marginally to the right. Therefore, there is no equilibrium in pure strategies such that $x=y \neq \frac{\beta}{\alpha+\beta}$.

If $\bar{x}=\frac{\beta}{\alpha+\beta}$ one can show using the same formal arguments as before that any deviation to the left or to the right of $\frac{\beta}{\alpha+\beta}$ benefits none of both players. That is, $\left(\frac{\beta}{\alpha+\beta}, \frac{\beta}{\alpha+\beta}\right)$ is the unique equilibrium of the game in pure strategies for these parameter values.

For this case, the unique pure strategy equilibrium that we identified is a quite robust prediction. Both due to the fact that the equilibrium is the unique in pure strategies and because it is symmetric coordination issues should not interfere with the result. Note that while the game is competitive, it need not be strictly competitive ${ }^{17}$ (Aumann 1961; Friedman 1983). To see this, consider that $\gamma=\delta=1$ and observe that $\pi_{1}(0,1)=\pi_{2}(0,1)=\frac{1}{2}$ while $\pi_{1}\left(\frac{\beta}{\alpha+\beta}, \frac{\beta}{\alpha+\beta}\right)=\pi_{2}\left(\frac{\beta}{\alpha+\beta}, \frac{\beta}{\alpha+\beta}\right)=\frac{\alpha \beta}{\alpha+\beta}<\frac{1}{2}$ as $\alpha+\beta>\alpha^{2}+\beta^{2} \geq$ $2 \alpha \beta$ when $0<\alpha, \beta<1$. This means that in such cases we have a prisoner's dilemma-like situation: a Nash equilibrium which is Pareto dominated. While not necessarily strictly competitive, the game still exhibits some competitive features and we conjecture that even in these cases there is no equilibrium in mixed strategies.

As far as comparative statics of this equilibrium are concerned we observe that the players' common location $\left(\frac{\beta}{\alpha+\beta}\right)$ is strictly decreasing (increasing) in $\alpha(\beta)$ but it is bounded from below by $\frac{1}{3}$. That is, in our two-player non-cooperative scenario, when centripetal forces are strong from both sides but somehow stronger from the left side, players converge to a moderate-left location $\left(\frac{\beta}{\alpha+\beta} \in\left(\frac{1}{3}, \frac{1}{2}\right]\right)$ as the peripheral voters' loyalty to the leftist party is greater. Finally, we note that in this case, candidates' location choices depend only on the values of $\alpha$ and $\beta$ and not on the exact voters' behavior in the central regions.

Case 2 - Mixed incentives $\left(\hat{\alpha}>\frac{1}{2}>\hat{\beta}\right)$.

In this case best response dynamics are non-trivial: if the first player is located to the left of the second one, then the first one has incentives to approach the second player while the second player has

\footnotetext{
${ }^{17} \mathrm{~A}$ game is strictly competitive if all possible outcomes are Pareto-optimal.
} 
incentives to move away from her opponent. These diverse incentives that players face rule out existence of Nash equilibria in pure strategies.

Proposition 3 If $\hat{\alpha}>\frac{1}{2}>\hat{\beta}$ then a) there is no pure strategy equilibrium and b) there exists a unique continuously differentiable atomless equilibrium $(G, G)$ with a convex support. When $2 \alpha+2 \beta \neq \gamma+\delta$ :

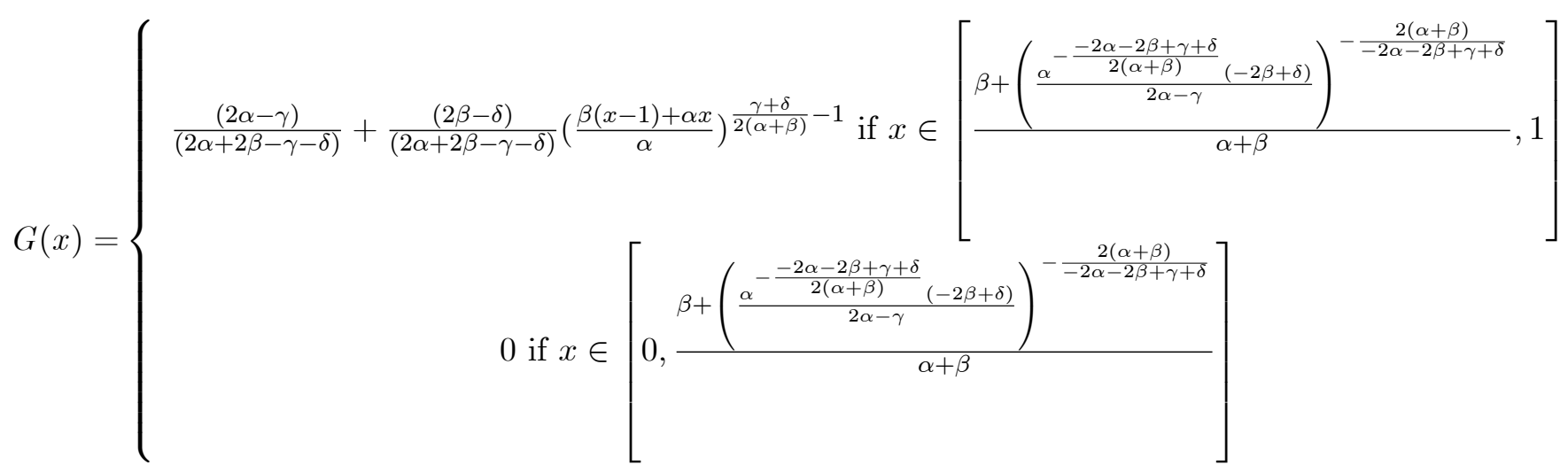

and when $2 \alpha+2 \beta=\gamma+\delta$ :

$$
G(x)=\left\{\begin{array}{c}
\frac{\gamma+\delta+(2 \beta-\delta)[\ln \alpha-\ln (\beta(x-1)+\alpha x)]}{\gamma+\delta} \text { if } x \in\left[\frac{\beta+\alpha e^{\frac{\gamma+\delta}{2 \beta-\delta}}}{\alpha+\beta}, 1\right] \\
0 \text { if } x \in\left[0, \frac{\beta+\alpha e^{\frac{\gamma+\delta}{2 \beta-\delta}}}{\alpha+\beta}\right]
\end{array}\right.
$$

Proof. Consider that the first player locates strictly to the right of the second player; $x<y$. Then:

$$
\frac{\partial \pi_{1}}{\partial x}(x, y)=\gamma\left(\hat{\alpha}-\frac{1}{2}\right)>0 \text { and } \frac{\partial \pi_{2}}{\partial y}(x, y)=\delta\left(\hat{\beta}-\frac{1}{2}\right)>0 .
$$

That is, the first player has incentives to approach the location of the second player and hence there is no pure strategy equilibrium in which players choose distinct locations.

Now consider that both players locate at the same interior point; $x=y=\bar{x} \in(0,1)$. In this case we have: 


$$
\pi_{1}(\bar{x}, \bar{x})=\pi_{2}(\bar{x}, \bar{x})=\frac{1}{2}(\beta(1-\bar{x})+\alpha \bar{x})
$$

As made clear by Remark 1 , for $(\bar{x}, \bar{x})$ to be an equilibrium it must be the case that $\bar{x}=\frac{\beta}{\alpha+\beta}$. At this point it holds that $\beta(1-\bar{x})=\alpha \bar{x}$. That is, for every positive $\alpha$ and $\beta$ this point is strictly smaller than one and since $\lim _{y \rightarrow \bar{x}^{+}} \pi_{2}(\bar{x}, y)=\beta(1-\bar{x})$ and $\frac{\partial \pi_{2}}{\partial y}(\bar{x}, y)>0$ for every $y>\bar{x}$, the second player has incentives to deviate to the right - no Nash equilibrium in pure strategies may exist.

If there exists a symmetric Nash equilibrium $(G, G)$ in mixed strategies such that $G$ is atomless and continuously differentiable with convex support $[\underline{g}, \bar{g}] \subseteq[0,1](G(\underline{g})=0$ and $G(\bar{g})=1)$, it must be the case that the first player is indifferent among all locations in $[\underline{g}, \bar{g}]$, that is:

$$
\pi_{1}(x, G)=\int_{\underline{g}}^{x}\left[\delta\left(x-\frac{x+y}{2}\right)+\beta(1-x)\right] d G(y)+\int_{x}^{\bar{g}}\left[\gamma\left(\frac{x+y}{2}-x\right)+\alpha x\right] d G(y)=\pi \text { for } x \in[\underline{g}, \bar{g}]
$$

and that she is never better off by locating out of $[\underline{g}, \bar{g}]$, that is, $\pi_{1}(x, G) \leq \pi$ for $x \notin[\underline{g}, \bar{g}]$. If there is such a $G$ then $\frac{\partial \pi_{1}}{\partial x}(x, G)=0$ for $x \in[\underline{g}, \bar{g}]$ which is equivalent (since $G$ is assumed to be continuously differentiable) to the differential equation:

$$
\frac{1}{2}(2 \alpha-\gamma)(1-G(x))-\frac{1}{2}(2 \beta-\delta) G(x)+\beta(1-x) G^{\prime}(x)-\alpha x G^{\prime}(x)=0 \text { for } x \in[\underline{g}, \bar{g}] .
$$

which has a unique solution up to a constant of integration. We notice that $\bar{g}$ must be equal to one. This is so because when $x>y$ we have that $\frac{\partial \pi_{1}}{\partial x}(x, y)=\gamma\left(\frac{1}{2}-\hat{\beta}\right)>0$. So if $\bar{g}<1$, then the first player has incentives to locate to the right of $\bar{g}$ because $\frac{\partial \pi_{1}}{\partial x}(x, G)=\gamma \int_{\underline{g}}^{\bar{g}}\left(\frac{1}{2}-\hat{\beta}\right) d G(y)=\left(\frac{1}{2}-\hat{\beta}\right)>0$ for any $x>\bar{g}$. That is, $\bar{g}$ is not a best response to $G$ and, thus, a symmetric continuously differentiable equilibrium $(G, G)$ with $\bar{g}<1$ is not possible. From this observation we get that $G(1)=1$. Using this information, we solve the above differential equation and we get the expression presented in the statement of the proposition.

Next we set $G(\underline{g})=0$ and we find: 


$$
\begin{aligned}
& \underline{g}=\frac{\beta+\left(\frac{\alpha^{-\frac{-2 \alpha-2 \beta+\gamma+\delta}{2(\alpha+\beta)}}(-2 \beta+\delta)}{2 \alpha-\gamma}\right)^{-\frac{2(\alpha+\beta)}{-2 \alpha-2 \beta+\gamma+\delta}}}{\alpha+\beta}>\frac{\beta}{\alpha+\beta}>0 \text { when } 2 \alpha+2 \beta \neq \gamma+\delta \text { and } \\
& \underline{g}=\frac{\beta+\alpha e^{\frac{\gamma+\delta}{2 \beta-\delta}}}{\alpha+\beta}>\frac{\beta}{\alpha+\beta}>0 \text { when } 2 \alpha+2 \beta=\gamma+\delta .
\end{aligned}
$$

We know that when $x<y$ we have that $\frac{\partial \pi_{1}}{\partial x}(x, y)=\gamma\left(\hat{\alpha}-\frac{1}{2}\right)>0$. So if $\underline{g}>0$ then $\frac{\partial \pi_{1}}{\partial x}(x, G)=$ $\gamma \int_{\underline{g}}^{1}\left(\hat{\alpha}-\frac{1}{2}\right) d G(y)=\gamma\left(\hat{\alpha}-\frac{1}{2}\right)>0$ for any $x<\underline{g}$. That is, if the second player plays $G$ then the first player strictly prefers $\underline{g}$ to any $x<\underline{g}$ and is indifferent among any of the locations in $[\underline{g}, 1]$; playing $G$ is a best response of the first player to the second player playing $G$. This concludes the argument.

It is important to note that the density is decreasing and therefore that the CDF is concave. Examples of such mixed strategies are provided on figure 2 .

\section{Insert Figure 2 here}

We have assumed $\hat{\alpha}>\frac{1}{2}>\hat{\beta}$, that is, strong centripetal incentives from the left and strong centrifugal incentives from the right. As we observe, the support of $G$ excludes locations at the extreme left but contains locations at the extreme right; the support of $G$ suggests that players will never locate at the extreme on whose side incentives are centripetal. Moreover, since both $\underline{g}$ and one belong to the support of $G$ it should hold that $\pi_{1}(\underline{g}, G)=\pi_{2}(1, G)$ and hence that $\alpha \underline{g}+\int_{\underline{g}}^{1} \gamma\left(\frac{y+\underline{g}}{2}-\underline{g}\right) d G(y)=\int_{\underline{g}}^{1} \delta \frac{1-y}{2} d G(y) \Leftrightarrow$ $\int_{0}^{1} y d G(y)=\frac{\delta-2 \alpha \underline{g}+\gamma \underline{g}}{\gamma+\delta}$. That is, the expected location of each player in this equilibrium depends on how voters behave both in central and in peripheral regions.

Case 3 - Centrifugal incentives $\left(\hat{\alpha} \leq \frac{1}{2}\right.$ and $\left.\hat{\beta}<\frac{1}{2}\right)$.

This case obviously corresponds to the opposite scenario of the first case. Here each player has incentives to move away from the other player when they find themselves located at distinct points. 
Proposition 4 If $\hat{\alpha} \leq \frac{1}{2}$ and $\hat{\beta}<\frac{1}{2}$, then there exists a unique piecewise continuously differentiable atomless equilibrium $(G, G)$ with a convex support. It is given by:

$$
G(x)=\left\{\begin{array}{l}
\frac{(2 \alpha-\gamma)}{(2 \alpha+2 \beta-\gamma-\delta)}-\frac{(2 \alpha-\gamma)}{(2 \alpha+2 \beta-\gamma-\delta)}\left(\frac{\beta(1-x)-\alpha x}{\beta}\right)^{\frac{\gamma+\delta}{2(\alpha+\beta)}-1} \text { if } x \in\left[0, \frac{\beta}{\alpha+\beta}\right) \\
\frac{(2 \alpha-\gamma)}{(2 \alpha+2 \beta-\gamma-\delta)}+\frac{(2 \beta-\delta)}{(2 \alpha+2 \beta-\gamma-\delta)}\left(\frac{\beta(x-1)+\alpha x}{\alpha}\right)^{\frac{\gamma+\delta}{2(\alpha+\beta)}-1} \text { if } x \in\left[\frac{\beta}{\alpha+\beta}, 1\right]
\end{array}\right.
$$

Proof. The derivation of the general form of $G(x)$ is performed as before. The big difference here is that, unlike the $\hat{\alpha}>\frac{1}{2}>\hat{\beta}$ case, the support $[\underline{g}, \bar{g}] \subseteq[0,1]$ of a symmetric atomless equilibrium in this case should be such that $[\underline{g}, \bar{g}]=[0,1]$. This is so because, if $\bar{g}<1$ (one can offer an equivalent argument to exclude the $\underline{g}>0$ case), then $\frac{\partial \pi_{1}}{\partial x}(x, G)=\gamma \int_{\underline{g}}^{\bar{g}}\left(\frac{1}{2}-\hat{\beta}\right) d G(y)=\gamma\left(\frac{1}{2}-\hat{\beta}\right)>0$ for any $x>\underline{g}$. That is, playing $\underline{g}$ is not a best response of the first player to the second player playing such a mixed strategy. Therefore, if a symmetric atomless equilibrium with convex support exists, it should satisfy $[\underline{g}, \bar{g}]=[0,1]$, $G(0)=0$ and $G(1)=1$. Since in this case $\hat{\alpha}+\hat{\beta} \neq 1$, the general form of $G(x)$ on any interval where $G$ is continuously differentiable is:

$$
G(x)=\frac{(2 \alpha-\gamma)}{(2 \alpha+2 \beta-\gamma-\delta)}+C(|\beta(1-x)-\alpha x|)^{\frac{\gamma+\delta}{2(\alpha+\beta)}-1}
$$

If we define:

$$
G_{A}(x)=\frac{(2 \alpha-\gamma)}{(2 \alpha+2 \beta-\gamma-\delta)}-\frac{(2 \alpha-\gamma)}{(2 \alpha+2 \beta-\gamma-\delta)}\left(\frac{\beta(1-x)-\alpha x}{\beta}\right)^{\frac{\gamma+\delta}{2(\alpha+\beta)}}-1
$$

and

$$
G_{B}(x)=\frac{(2 \alpha-\gamma)}{(2 \alpha+2 \beta-\gamma-\delta)}+\frac{(2 \beta-\delta)}{(2 \alpha+2 \beta-\gamma-\delta)}\left(\frac{\beta(x-1)+\alpha x}{\alpha}\right)^{\frac{\gamma+\delta}{2(\alpha+\beta)}-1}
$$

we observe that $G_{A}(x)=G_{B}(x)$ if and only if $x=\frac{\beta}{\alpha+\beta}$. Moreover we have that $G_{A}(0)=0, \frac{\partial G_{A}}{\partial x}>0$ for any $x \in\left[0, \frac{\beta}{\alpha+\beta}\right], \frac{\partial G_{B}}{\partial x}>0$ for any $x \in\left[\frac{\beta}{\alpha+\beta}, 1\right]$ and $G(1)=1$. In other words $G$, as defined in 
the statement of this proposition, is a piecewise continuously differentiable strictly increasing cumulative distribution function with full support in $[0,1]$.

So if the first player uses this strategy then $\pi_{2}(G, y)=\bar{\pi}$ for any $y \in[0,1]-G$ is a best response of the second player to the first player playing $G$.

Now assume that there exists another piecewise continuously differentiable $\hat{G} \neq G$ which defines another atomless symmetric equilibrium. Then, the first piece of $\hat{G}$ should also be identical to $G_{A}$ because, otherwise, $\hat{G}$ would not be equal to zero for $x=0$ and strictly positive for every $x>0$; and, hence, $\hat{G}$ would either have an atom at zero (and, therefore, it would not define an atomless equilibrium) or its support would not coincide with $[0,1]$ (this contradicts the arguments in the beginning of this proof). Moreover, $\hat{G}$ may not have a piece distinct to $G_{A}$ which starts before $\frac{\beta}{\alpha+\beta}$. If this were true $\hat{G}$ would have a discontinuity at the point at which the new piece starts; and this would contradict the fact that it defines an atomless equilibrium. This is so because:

$$
\frac{(2 \alpha-\gamma)}{(2 \alpha+2 \beta-\gamma-\delta)}+C^{\prime}(|\beta(1-x)-\alpha x|)^{\frac{\gamma+\delta}{2(\alpha+\beta)}-1} \neq \frac{(2 \alpha-\gamma)}{(2 \alpha+2 \beta-\gamma-\delta)}+C^{\prime \prime}(|\beta(1-x)-\alpha x|)^{\frac{\gamma+\delta}{2(\alpha+\beta)}-1}
$$

for every $x<\frac{\beta}{\alpha+\beta}$ when $C^{\prime} \neq C^{\prime \prime}$.

Equivalently, we can show that $\hat{G}$ may not have a piece distinct to $G_{B}$ which ends after $\frac{\beta}{\alpha+\beta}$. Therefore, $G$ must be the unique piecewise continuously differentiable function that defines a symmetric atomless equilibrium.

To discuss some of the qualitative features of this equilibrium we focus on the case in which $\gamma=\delta=1$. That is, in the case in which all voters located in the central regions vote for one of the two players. Different shapes of the density may appear, as illustrated on figure 3.

\section{Insert Figure 3 here}

If $\alpha+\beta$ is less than $\frac{1}{2}$, the probability mass will be more on the extremes with a density first decreasing and then increasing (figures $3 a$ and $3 b$ ). If $\alpha+\beta=\frac{1}{2}$, then $G$ is uniform (figure $3 c$ ). Finally, if $\alpha+\beta$ is 
larger than $\frac{1}{2}$, then the probability mass is more concentrated in the center with a density first increasing and then decreasing (figure $3 d$ ).

Remark 2 There is a smooth transition from the pure symmetric Nash equilibria of Proposition 2 to the mixed ones of Proposition 4. For the sake of illustration take $\alpha=\beta$ and $\gamma=\delta=1$. When $\alpha \in\left(0, \frac{1}{4}\right]$, the mass of $\frac{1}{2}$ on both sides of the center of the policy space is located around the two extremes, and tends to $\frac{1}{2} \zeta_{0}+\frac{1}{2} \zeta_{1}{ }^{18}$ when $\alpha$ tends to 0 . That is, when $(\alpha, \beta)$ converges to $(0,0)$ a symmetric mixed equilibrium of the game converges to the diagonal profile of mixed strategies $(G, G)$ where $G=\frac{1}{2} \zeta_{0}+\frac{1}{2} \zeta_{1}$. In contrast, when $\alpha \in\left(\frac{1}{4}, \frac{1}{2}\right]$, the mass of $\frac{1}{2}$ on both sides of $\frac{1}{2}$ is located around $\frac{1}{2}$ and tends to $\zeta_{\frac{1}{2}}$ when $\alpha$ tends to $\frac{1}{2}$.

Since both zero and one belong to the support of $G$ it should hold that $\pi_{1}(0, G)=\pi_{2}(1, G)$ and hence that $\int_{0}^{1} \gamma \frac{y}{2} d G(y)=\int_{0}^{1} \delta \frac{1-y}{2} d G(y) \Leftrightarrow \int_{0}^{1} y d G(y)=\frac{\delta}{\gamma+\delta}$. That is, the expected location of each player in this equilibrium depends only on how voters in the central regions behave.

To sum up we have that when centrifugal forces are very weak from both sides, expected equilibrium location depends only on how voters in the peripheral regions behave; when centrifugal forces are strong from on side and weak from the other, then expected equilibrium location is sensitive on how voters in both kinds of regions behave, and, finally, when centrifugal forces are strong on both sides, expected equilibrium location depends only on how voters in central regions behave.

When centrifugal incentives dominate on both sides there also exist two asymmetric pure strategy equilibria and many asymmetric equilibria in mixed strategies.

Proposition 5 If $\hat{\alpha}, \hat{\beta}<\frac{1}{2}$ then the pure strategy profiles $(0,1)$ and $(1,0)$ are the unique pure strategy equilibria of the game.

Proof. Consider that the first player locates strictly to the left of the second player; $x<y$. Then:

$$
\frac{\partial \pi_{1}}{\partial x}(x, y)=\gamma\left(\hat{\alpha}-\frac{1}{2}\right)<0 \text { and } \frac{\partial \pi_{2}}{\partial y}(x, y)=\delta\left(\frac{1}{2}-\hat{\beta}\right)>0 .
$$

\footnotetext{
${ }^{18} \mathrm{By} \zeta_{z}$ we denote the Dirac mass in $z$.
} 
That is, if there are pure strategy equilibria such that players locate at distinct locations, then they should be such that $x=0$ and $y=1$ or $x=1$ and $y=0$. To validate that these strategy profiles are indeed equilibria we only have to show that when one player locates at one extreme, then the other player prefers to locate at the opposite extreme rather than to the same as her opponent. We notice that $\pi_{1}(0,1)=\pi_{2}(1,0)=\frac{\gamma}{2}>\frac{\alpha}{2}=\pi_{1}(1,1)=\pi_{2}(1,1)$ and hence when the second (first) player locates at the right extreme, the unique best response of the first (second) player is to locate to the left extreme. In a similar manner one can establish that when one player locates at the left extreme the unique best response of the other player is to locate at the right extreme.

Finally, from remark 1 we know that if there is a convergent pure strategy equilibrium it should be such that both players locate at $\frac{\beta}{\alpha+\beta}$. Given that the payoff function of the first player is continuous in own strategy when the second player locates at $\frac{\beta}{\alpha+\beta}$ and that $\frac{\partial \pi_{1}}{\partial x}\left(x, \frac{\beta}{\alpha+\beta}\right)<0(>0)$ for any $x<\frac{\beta}{\alpha+\beta}$ $\left(x<\frac{\beta}{\alpha+\beta}\right)$ it follows that we cannot have convergent pure strategy equilibria in this case.

One can further show that a variety of asymmetric mixed equilibria exists when centrifugal incentives dominate in both directions. To demonstrate this, we consider for simplicity that $\gamma=\delta=1$ and we construct asymmetric equilibria of the game. Let $R$ be the mixed strategy $p \zeta_{0}+(1-p) \zeta_{1}$ where $p \in[0,1]$. Since $\pi_{1}(x, R)=p\left(\frac{x}{2}+\beta(1-x)\right)+(1-p)\left(\alpha x+\frac{1-x}{2}\right)$ for all $x \in(0,1)$, we obtain that it does not depend upon $x$ if and only if:

$$
p=\frac{1}{2} \times \frac{1-2 \alpha}{1-\beta-\alpha}
$$

In such cases, since $\pi_{1}(0, R)=p \frac{\beta}{2}+(1-p) \frac{1}{2}$ and $\pi_{1}(1, R)=p \frac{1}{2}+(1-p) \frac{\alpha}{2}$ are strictly smaller than $\pi_{1}(x, R)$ for all $x \in(0,1)$, we obtain that any $x \in(0,1)$ is a best response to $R$. In particular, $\frac{1}{2}$ is a best response to $R$. Since both 0 and 1 are best responses to $\frac{1}{2}$, we have demonstrated that $\left(\frac{1}{2}, R\right)$ and $\left(R, \frac{1}{2}\right)$ are Nash equilibria. Consider also as before the off-diagonal profile $(U, R)$ where $U$ denotes the uniform probability on $[0,1]$. What is the best response of player 2 to $U$ ? Since $\pi_{2}(U, y)=$ $\left(\frac{1}{2}-\alpha-\beta\right) y^{2}+\left(\alpha+\beta-\frac{1}{2}\right) y+\frac{1}{4}$ is convex and symmetric around $\frac{1}{2}$ if $\alpha=\beta$ and $\alpha<\frac{1}{4}, 0$ and 1 are the best responses. Therefore, if $\alpha=\beta$ and $\alpha<\frac{1}{4},(U, R)$ is also a Nash equilibrium. Finally, consider the strategy profile $(G, R)$ where $G$ is the absolutely continuous mixed strategy identified in Proposition 4 . We argued that any $x \in(0,1)$ is a best response to $R$ and, thus, $G$ is a best response to $R$. Moreover we know that any $y \in[0,1]$ is a best response to $G$. That is, $(G, R)$ is also an equilibrium for $(\alpha, \beta) \in\left(0, \frac{1}{2}\right)^{2}$. 


\section{Concluding Remarks}

We have analyzed equilibrium behavior in a general spatial competition model which we believe to be relevant especially in electoral competition. Our results indicate that an equilibrium exists in the spatial competition model for a general class of centrifugal incentives (essentially any centrifugal incentives that may arise due to partial voter participation). We moreover show that standard convergent equilibrium behavior is robust to introducing low to mild centrifugal forces in one or both directions but it collapses once centrifugal forces become strong even in only one direction. Analysis of a symmetric equilibrium for the simplest parametrization of our model (constant functions and uniform distribution) gives us a hint of what are most probable outcomes of such games and proves that, in this model, there are no sudden changes in equilibrium behavior when parameter values change smoothly despite the fact that we may have transitions from equilibria in pure strategies to ones in mixed strategies.

\section{References}

[1] Adams, J. F., Merrill III, S. and B. Grofman (2005). A unified theory of party competition: a crossnational analysis integrating spatial and behavioral factors. Cambridge University Press.

[2] Aragonès, E. and T. Palfrey (2002). Mixed strategy equilibrium in a Downsian model with a favored candidate. Journal of Economic Theory, 103,131-161.

[3] Aragonès, E. and D. Xefteris (2013). Imperfectly informed voters and strategic extremism. Barcelona GSE Working Paper Series, 725.

[4] Aumann, R.J (1961). Almost Strictly Competitive Games. Journal of the Society for Industrial and Applied Mathematics, 9, 544-550.

[5] Cancian M., Bergström T. and A. Bills (1995). Hotelling Location Problems with Directional Constraints: An Application to Television News Scheduling. Journal of Industrial Economics, 43, 121-124.

[6] Cox, G.W. (1990). Centripetal and Centrifugal Incentives in Electoral Systems. American Journal of Political Science, 34, 903-935.

[7] Dasgupta, P. and E. Maskin (1986) .The Existence of Equilibrium in Discontinuous Games. Review of Economic Studies, 53, 1-26. 
[8] Downs, A. (1957). An Economic Theory of Democracy. New York, Harper and Row.

[9] Duggan, J. (2012). A Survey of Equilibrium Analysis in Spatial Models of Elections. University of Rochester, Mimeo.

[10] Fey, M. (2012). Symmetric games with only asymmetric equilibria. Games and Economic Behavior, $75(1), 424-427$.

[11] Friedman, J.W. (1983). On Characterizing Equilibrium Points in Two Person Strictly Competitive Games. International Journal of Game Theory, 12, 245-247.

[12] Gabszewicz, J., Laussel, D. and M. Le Breton (2008). The Mixed Strategy Nash Equilibrium of the Television News Scheduling Game. Journal of Industrial Economics, 56, 418-425.

[13] Glazer, A., Grofman, B. and G. Owen (1998). A neo-Downsian model of group-oriented voting and racial backlash. Public Choice, 97, 23-34.

[14] Groseclose, T. (2001). A Model of Candidate Location When One Candidate Has a Valence Advantage. American Journal of Political Science, 45, 862-86.

[15] Hortala-Vallve, R. and B. Esteve-Volart (2011). Voter turnout and electoral competition in a multidimensional policy space. European Journal of Political Economy, 27(2), 376-384.

[16] Hotelling, H. (1929). Stability in Competition. Economic Journal. 39, 41-57.

[17] Indridason, I. H. (2013). Expressive motives and third-party candidates. Journal of Theoretical Politics, 25(2), 182-213.

[18] Laussel, D. and M. Le Breton (2002). Unidimensional Downsian Politics: Median, Utilitarian, or What Else? Economics Letters, 76, 351-356.

[19] Matakos, K., Troumpounis, O. and D. Xefteris, D. (2015a). Electoral Rule Disproportionality and Platform Polarization. American Journal of Political Science, doi: 10.1111/ajps.12235.

[20] Matakos, K., Troumpounis, O. and D. Xefteris (2015b). Turnout and polarization under alternative electoral systems. The Political Economy of Governance, Eds. N. Schofield and G. Caballero, 335-362. Springer International Publishing.

[21] Merrill III, S. and J. Adams (2002). Centrifugal incentives in multicandidate elections. Journal of Theoretical Politics, 14, 275-300. 
[22] Osborne, M. (1995). Spatial Models of Political Competition Under Plurality Rule: A Survey of Some Explanations of the Number of Candidates and the Positions They Take. Canadian Journal of Economics, 28, 261-301.

[23] Roemer, J. (2001) Political Competition: Theory and Applications, Cambridge, Harvard University Press.

[24] Xefteris, D. (2013). Equilibria in unidirectional spatial models. Economics Letters, 119:2, 146-149.

[25] Xefteris, D. (2015). Symmetric zero-sum games with only asymmetric equilibria. Games and Economic Behavior, 89, 122-125.

\section{Appendix}

In this appendix we provide some discussion of pure strategy equilibria when the distribution of voters in nonuniform and some microfoundations of the constant functions' quadruple $(\alpha, \beta, \gamma, \delta)$ that was extensively used throughout the paper.

\subsection{Nonuniform distributions}

In the case of a uniform distribution, when $\hat{\alpha}=\hat{\beta}$, the game admits Nash equilibria in pure strategies: $\left(\frac{1}{2}, \frac{1}{2}\right)$, no differentiation, when $\hat{\alpha}>\frac{1}{2}$ and $(0,1)$, maximal differentiation, when when $\hat{\alpha}<\frac{1}{2}$. The existence of a local ${ }^{19}$ Nash equilibrium exhibiting no differentiation when $\hat{\alpha}>\frac{1}{2}$ and differentiation when $\hat{\alpha}<\frac{1}{2}$ continues to hold for a large class of distributions. Consider a distribution described by the density $f$ which will be assumed differentiable, symmetric with respect to $\frac{1}{2}$ (that is, $f(x)=f(1-x)$ for all $x \in\left[0, \frac{1}{2}\right]$ ) and strictly increasing on $\left[0, \frac{1}{2}\right]$ (and so strictly decreasing on $\left[\frac{1}{2}, 1\right]$ ). Let $(x, y)$ be a profile of locations such that: $x<\frac{1}{2} \leq y$. Without loss of generality ${ }^{20}$ assume that $\frac{x+y}{2} \leq \frac{1}{2}$. For the player located on the left, moving on the right leads to a gain of $\frac{\gamma}{2} f\left(\frac{x+y}{2}\right)$ and to a loss of $\gamma(1-\hat{\alpha}) f(x)$. If $\hat{\alpha} \geq \frac{1}{2}$, then the gain is always larger than the cost and the equilibrium is defined by $x=y=\frac{1}{2}$. If otherwise $\hat{\alpha}<\frac{1}{2}$, a marginal equilibrium is obtained when the marginal rate of substitution $\frac{\frac{1}{2} f\left(\frac{x+y}{2}\right)}{(1-\hat{\alpha})) f(x)}$ is equal to 1 , that is,

\footnotetext{
${ }^{19}$ We have not investigated the general conditions on $f$ under which this LNE is a (global) Nash equilibrium.

${ }^{20}$ If $\frac{x+y}{2} \geq \frac{1}{2}$, conduct the same argument from the perspective of the player on the right.
} 


$$
\frac{1}{2} f\left(\frac{x+y}{2}\right)=(1-\hat{\alpha}) f(x)
$$

or

$$
f(x)=\frac{1}{2(1-\hat{\alpha})} f\left(\frac{x+y}{2}\right)
$$

Let us test when the symmetric profile $(x, 1-x)$ with $x<\frac{1}{2}$ is a (local) Nash equilibrium. ${ }^{21}$ From above, the first order condition writes:

$$
f(x)=\frac{1}{2(1-\hat{\alpha})} f\left(\frac{1}{2}\right)
$$

If $f(0)=0$, then the above equation has a unique solution $x^{*}$. We may also check that the (local) second order condition is satisfied. Indeed the second derivative at $x^{*}$

$$
\frac{1}{4} f^{\prime}\left(\frac{1}{2}\right)+(\hat{\alpha}-1) f^{\prime}\left(x^{*}\right)=(\hat{\alpha}-1) f^{\prime}\left(x^{*}\right)
$$

is negative as $\hat{\alpha}<1$. For the sake of illustration, consider the (symmetric) Beta distribution ${ }^{22}$ :

$$
f(x)=\frac{\Gamma(2 \xi)}{\Gamma(\xi)^{2}}(x(1-x))^{\xi-1} \text { over }[0,1]
$$

with $\xi>1$. In such case, $x^{*}$ is the solution of the equation:

$$
x(1-x)=\frac{1}{4}\left(\frac{1}{2(1-\hat{\alpha})}\right)^{\frac{1}{\xi-1}}
$$

We obtain:

\footnotetext{
${ }^{21}$ Since we have assumed $f$ strictly increasing, this argument does not apply to the uniform distribution. In fact, for the uniform distribution the marginal rate of substitution at any profile $(x, y)$ is equal to $\frac{1}{2(1-\hat{\alpha})}$.

${ }^{22} \Gamma$ denotes the Gamma function. In particular $\Gamma(n)=(n-1)$ ! for any integer $n$.
} 


$$
x^{*}=\frac{1}{2}-\frac{\sqrt{1-\left(\frac{1}{2(1-\hat{\alpha})}\right)^{\frac{1}{\xi-1}}}}{2}
$$

As we observe differentiation (defined by $1-2 x^{*}(\hat{\alpha}, \xi)$ ) is reduced when $F$ is more concentrated around the center (large values of $\xi$ ) and when the centrifugal incentives are less intense (large values of $\hat{\alpha}$ ). This is illustrated on table 1 for $\hat{\alpha}=0$ and on table 2 for $\hat{\alpha}=\frac{1}{4}$.

$\begin{array}{cccc}\xi & 2 & 10 & 100 \\ x^{*} & 0.14645 & 0.36387 & 0.45824\end{array}$

Table 1

$\begin{array}{cccc}\xi & 2 & 10 & 100 \\ x^{*} & 0.21132 & 0.39506 & 0.46803\end{array}$

Table 2

\subsection{An application}

In this section we develop a model of electoral competition among four parties, two instrumental and two parametric ones, and we demonstrate that it is a particular case of the general model studied above. Let $\{l, 1,2, r\}$ be the set of political parties and $[0,1]$ the policy space. The policy platforms of the extremist parties, $l$ and $r$, are fixed exogenously and are 0 and 1 respectively. ${ }^{23}$ Parties 1 and 2 - which we call mainstream - strategically decide their policy platforms, $x$ and $y$, in order to maximize their vote-share and voters' ideal policies are distributed uniformly on the interval $[0,1]$.

We consider a two-stage extended form game of perfect information and, naturally, the solution concept that we apply is subgame perfection. In the first stage, the two mainstream parties decide their policy platforms and then the voters observe $(x, y) \in[0,1]^{2}$ and they vote. We allow both pure and mixed strategies for any of our players. Let $\left(\sigma^{1}, \sigma^{2}\right)$ denote a pair of mixed strategies for the two mainstream candidates (probability distributions such that their support is a subset of $[0,1])$ and $\hat{\sigma}^{i}(x, y)$ denote a

\footnotetext{
${ }^{23}$ Another example of an electoral competition model with non-instrumental extremist parties is Indridason (2013).
} 
mixed strategy of a voter with ideal policy $i \in[0,1]$ (probability distribution such that its support is a subset of $\{l, 1,2, r\})$ when the voter observes that the policy platforms of the two mainstream parties are given by $(x, y) \in[0,1]^{2}$. Then a strategy profile in our game is given by $\sigma=\left\{\left(\sigma^{1}, \sigma^{2}\right), \hat{\sigma}\right.$ such that $\hat{\sigma}(x, y) \in \hat{\sigma}$ for all $\left.(x, y) \in[0,1]^{2}\right\}$ where $\hat{\sigma}(x, y)$ is such that $\hat{\sigma}^{i}(x, y) \in \hat{\sigma}(x, y)$ for every $i \in[0,1]$ and each possible subgame $(x, y) \in[0,1]^{2}$.

Each extremist/niche party is assumed to promote a cause that is independent of the main policy issue and which voters' may find attractive or repulsive. ${ }^{24}$ Voters' valuation of a cause promoted by a party is captured by an attraction/repulsion parameter $\phi_{k} \in \mathbb{R}$ for $k \in\{l, 1,2, r\}$. If $\phi_{k}>0$ then the promoted goal is attractive and if $\phi_{k}<0$ it is repulsive (we assume that mainstream parties do not promote such causes and hence $\left.\phi_{1}=\phi_{2}=0\right)$.

Our voters are expressive and sophisticated at the same time; they know that they cannot individually influence the outcome of elections - hence they derive utility only from voting for the party that they prefer - but they are able to take in account expectations about the voting behavior of their fellow citizens whenever this is relevant. We consider that the payoff of a voter with ideal policy $i \in[0,1]$ who votes for a party $k \in\{l, 1,2, r\}$ with policy platform $\psi_{k} \in[0,1]$ when we are in subgame $(x, y) \in[0,1]^{2}$ and all other voters are expected to behave according to $\hat{\sigma}^{-i}(x, y)=\hat{\sigma}(x, y)-\left\{\hat{\sigma}^{i}(x, y)\right\}$ is given by:

$$
u_{i}\left(\psi_{k}, \phi_{k}, \hat{\sigma}^{-i}(x, y)\right)=-\left|\psi_{k}-i\right|+\phi_{k} \times v_{k}\left(\hat{\sigma}^{-i}(x, y)\right)
$$

where $v_{k}\left(\hat{\sigma}^{-i}(x, y)\right)$ is the expected vote-share of party $k \in\{l, 1,2, r\}$ when all other voters are expected to vote according to $\hat{\sigma}^{-i}(x, y)$.

Notice that the component of the above utility function which does not relate to the position of the party on the main political issue is not fixed ${ }^{25}$ as in Groseclose (2001) or Aragonès and Palfrey (2002) but it also depends on the "electoral power" of each party. This is so because, if the cause that a niche party $k \in\{l, r\}$ promotes is attractive for the voters then the impact of this niche party on government policy outputs - and hence on the voters welfare - will be increasing in the niche party's vote-share and vice versa.

Consider that we are in subgame $(x, y) \in[0,1]^{2}$ with $x<y$ and that $\hat{\sigma}(x, y)$ is such that both

\footnotetext{
${ }^{24}$ We also have results, which are available upon request, for a case in which voters' are allowed to have heterogeneous valuations on the goal promoted by an extremist/niche party and which are in line with the present analysis.

${ }^{25}$ Glazer, Grofman and Owen (1998) also consider voters who evaluate a party differently depending on who else is expected to vote for it. In their case the externality is not anonymous while in our case it is.
} 
mainstream parties get a strictly positive vote-share. Then, if $\hat{\sigma}(x, y)$ is a Nash equilibrium of the $(x, y) \in$ $[0,1]^{2}$ subgame there should exist $i_{l 1} \in[0, x]$ such that: ${ }^{26}$

$$
u_{i_{l 1}}\left(0, \phi_{l}, \hat{\sigma}^{-i_{l 1}}(x, y)\right)=u_{i_{l 1}}\left(x, \phi_{1}, \hat{\sigma}^{-i_{l 1}}(x, y)\right)
$$

which is equivalent to

$$
i_{l 1}=\frac{1}{2-\phi_{l}} x
$$

because when $F$ is uniform, $x<y$ and $\hat{\sigma}(x, y)$ is a Nash equilibrium of the $(x, y) \in[0,1]^{2}$ subgame such that all parties get a strictly positive vote-share, it must be the case that $v_{l}\left(\hat{\sigma}^{-i_{l 1}}(x, y)\right)=i_{l 1}$.

Equivalently, one can show that in a Nash equilibrium of the $(x, y) \in[0,1]^{2}$ subgame in which all parties get positive vote-shares there should also exist $i_{12} \in[x, y]$ and $i_{2 r} \in[y, 1]$ such that:

$$
u_{i_{12}}\left(x, \phi_{1}, \hat{\sigma}^{-i_{12}}(x, y)\right)=u_{i_{12}}\left(y, \phi_{2}, \hat{\sigma}^{-i_{12}}(x, y)\right)
$$

and

$$
u_{i_{2 r}}\left(y, \phi_{2}, \hat{\sigma}^{-i_{2 r}}(x, y)\right)=u_{i_{2 r}}\left(1, \phi_{r}, \hat{\sigma}^{-i_{2 r}}(x, y)\right)
$$

which are equivalent to

$$
i_{12}=\frac{x+y}{2}
$$

and

$$
i_{2 r}=\frac{1+y-\phi_{r}}{2-\phi_{r}}
$$

\footnotetext{
${ }^{26}$ It is worth mentioning that in an equilibrium of an $(x, y) \in[0,1]^{2}$ subgame in which both mainstream parties get a positive vote-share it should be the case that centrist voters (voters with ideal policies between $x$ and $y$ ) never vote for extremist parties. This is so because if $u_{i}\left(0, \theta_{l}, \hat{\sigma}^{-i}(x, y)\right)>u_{i_{l}}\left(x, \theta_{1}, \hat{\sigma}^{-i}(x, y)\right)$ for some $i \in[x, y]$ then it should also hold that every voter prefers party $l$ to party 1 and hence, the vote-share of party 1 must be zero.
} 
It is straightforward that if the attraction parameter of the leftist niche party is sufficiently large $\left(\phi_{l} \geq 1\right)$ then in any Nash equilibrium of any subgame $(x, y) \in[0,1]^{2}$, it should be the case that no mainstream party gets any votes and, hence, our game becomes trivial: the mainstream parties are indifferent among all available strategies. This obviously holds for the attraction parameter of the rightist niche party too. Therefore, we focus on attraction parameters which make our game non-trivial: $\phi_{l}, \phi_{r}<1$.

If the attraction parameters of the niche parties are small enough, $\phi_{l}, \phi_{r}<1$, then for every subgame $(x, y) \in[0,1]^{2}$ there exists a unique Nash equilibrium such that both mainstream parties get a positive vote-share. The payoff of mainstream party 1 in this Nash equilibrium of any $(x, y) \in[0,1]^{2}$ subgame is given by:

$$
\pi_{1}(x, y)=\left\{\begin{array}{l}
x\left(\frac{1-\phi_{l}}{2-\phi_{l}}\right)+\frac{y-x}{2} \text { if } 0 \leq x<y \leq 1 \\
\frac{1}{2}\left(x \frac{1-\phi_{l}}{2-\phi_{l}}+(1-x) \frac{1-\phi_{r}}{2-\phi_{r}}\right) \text { if } x=y \\
(1-x) \frac{1-\phi_{r}}{2-\phi_{r}}+\frac{x-y}{2} \text { if } 0 \leq y<x \leq 1
\end{array}\right.
$$

and the payoff of mainstream party 2 is given by:

$$
\pi_{2}(x, y)=\left\{\begin{array}{l}
y\left(\frac{1-\phi_{l}}{2-\phi_{l}}\right)+\frac{x-y}{2} \text { if } 0 \leq y<x \leq 1 \\
\frac{1}{2}\left(y \frac{1-\phi_{l}}{2-\phi_{l}}+(1-y) \frac{1-\phi_{r}}{2-\phi_{r}}\right) \text { if } y=x \\
(1-y) \frac{1-\phi_{r}}{2-\phi_{r}}+\frac{y-x}{2} \text { if } 0 \leq x<y \leq 1
\end{array}\right.
$$

Hence, this two player game is identical to the one we extensively analyzed in the previous section for $\alpha=\frac{1-\phi_{l}}{2-\phi_{l}}, \beta=\frac{1-\phi_{r}}{2-\phi_{r}}$ and $\gamma=\delta=1$. When both extremist parties promote repulsive causes $\phi_{l}, \phi_{r}<1$ (strong centripetal incentives from both sides) we have a unique convergent equilibrium to the side of the more repulsive extremist party, when one extremist party promotes a repulsive cause and the other extremist party promotes an attractive cause (mixed incentives) then we have a symmetric equilibrium in mixed strategies such that mainstream parties locate (in expected terms) to the side of the extremist party which promote the repulsive cause and when both extremist parties promote attractive causes, there is a symmetric equilibrium in mixed strategies such that the mainstream parties locate (in expected terms) in the center of the policy space. 
$\pi_{1}(x, y)$

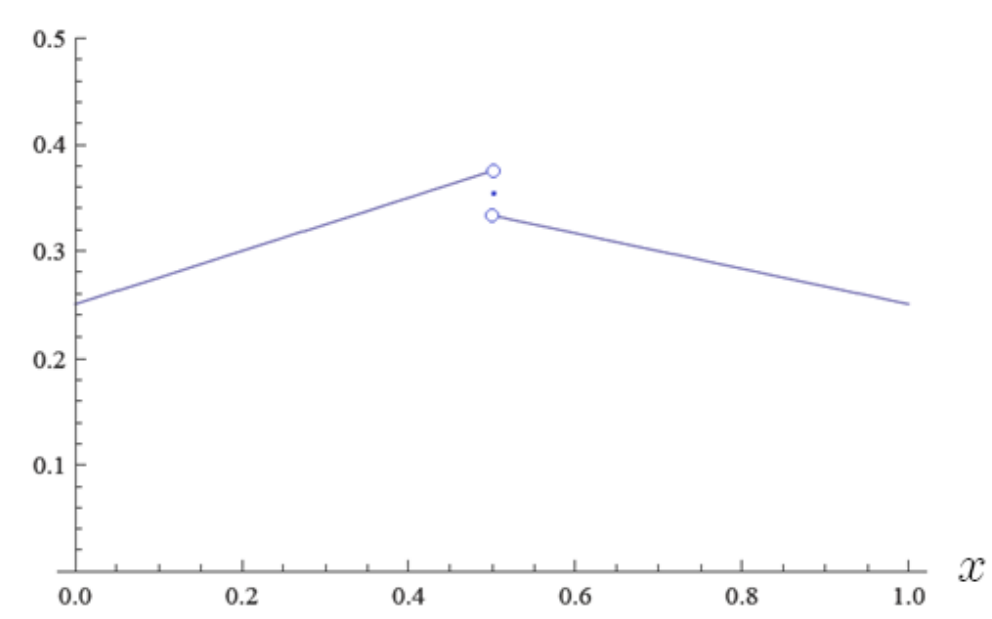

a) $\alpha=3 / 4, \beta=2 / 3, \gamma=\delta=1$ and $y=1 / 2$

$$
\pi_{1}(x, y)
$$

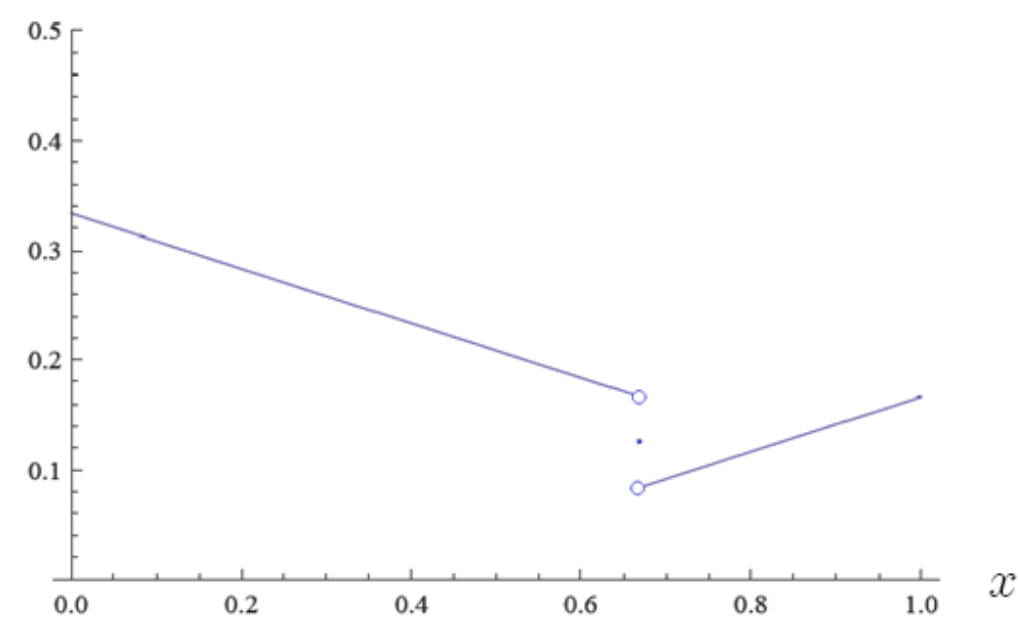

c) $\alpha=1 / 4, \beta=1 / 4, \gamma=\delta=1$ and $y=2 / 3$

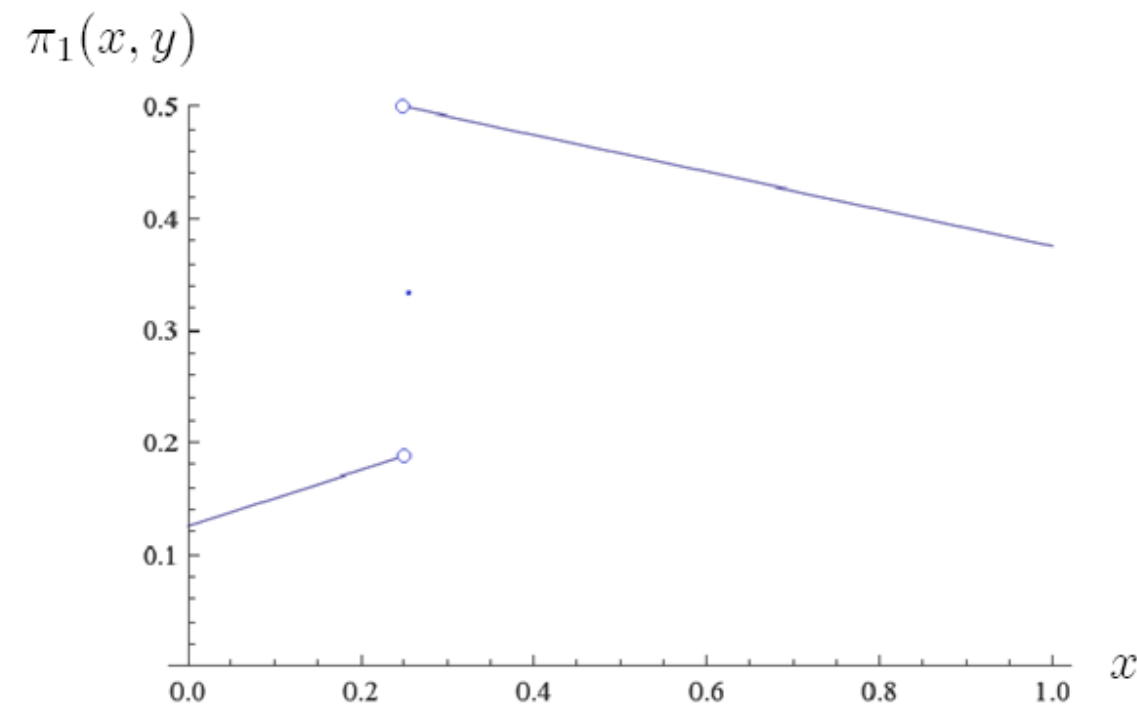

b) $\alpha=3 / 4, \beta=2 / 3, \gamma=\delta=1$ and $y=1 / 4$

$\pi_{1}(x, y)$

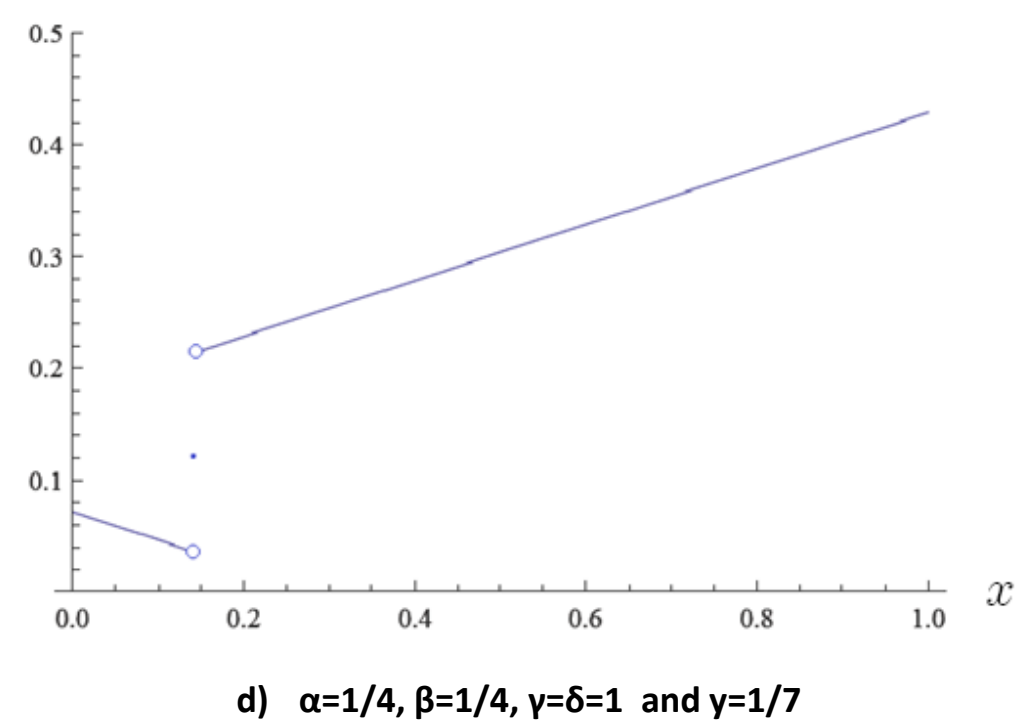




$$
\pi_{1}(x, y)
$$

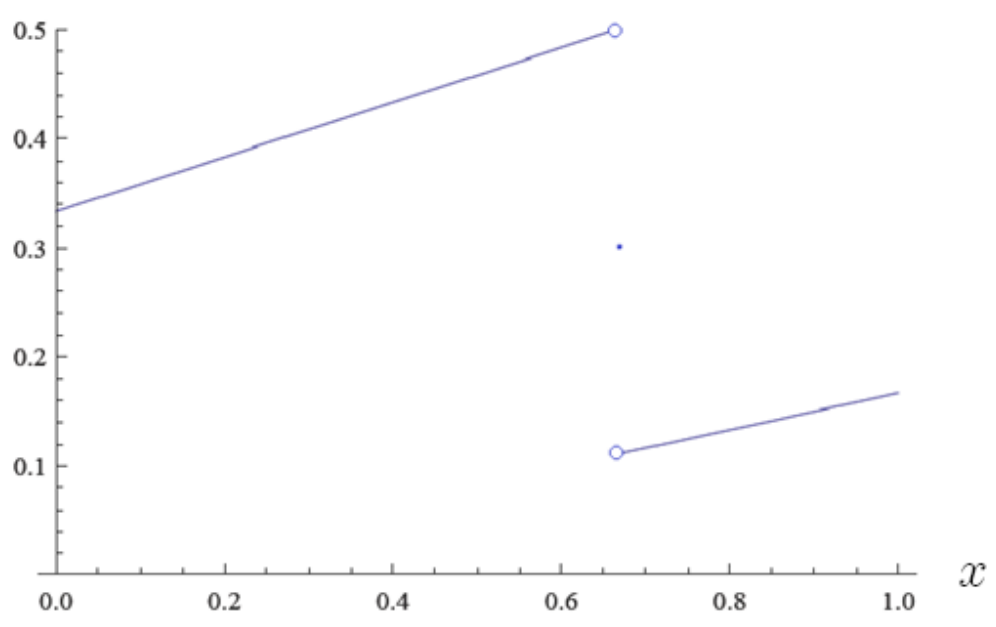

e) $\alpha=3 / 4, \beta=1 / 3, \gamma=\delta=1$ and $y=2 / 3$ $\pi_{1}(x, y)$

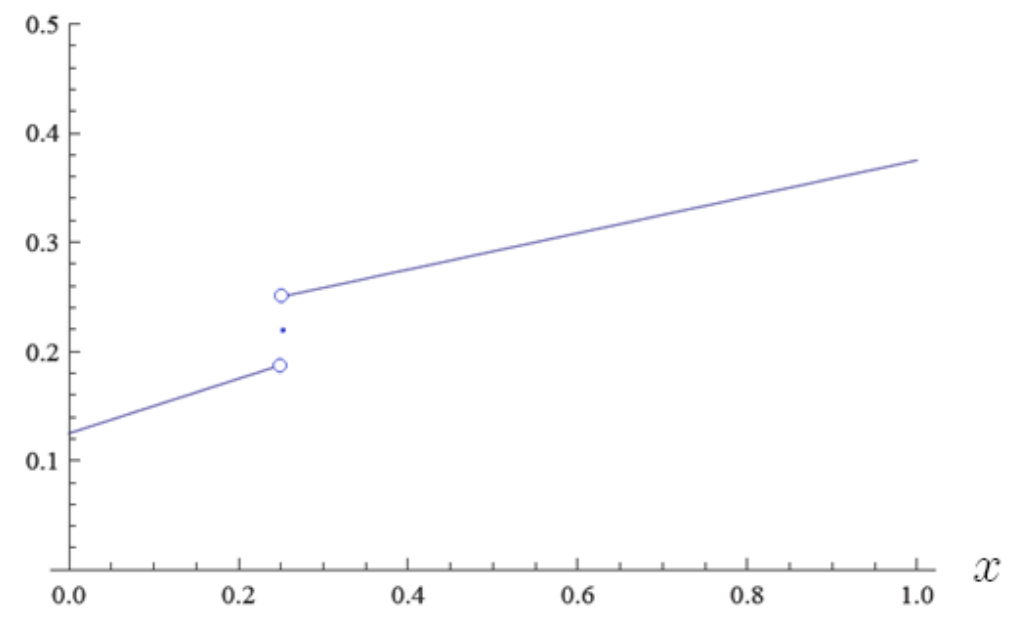

f) $\alpha=3 / 4, \beta=1 / 3, \gamma=\delta=1$ and $y=1 / 4$

Figure 1. The payoff of the first player as a function of $x$ for various values of $\alpha, \beta$ and $y$. 


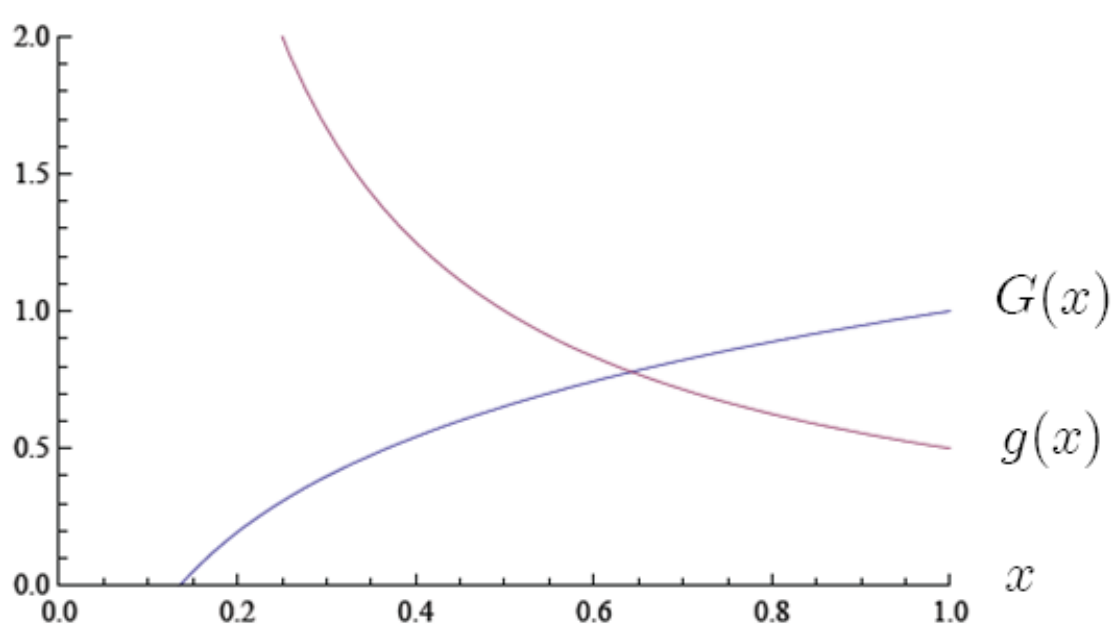

a) $\alpha \rightarrow 1$ and $\beta \rightarrow 0$

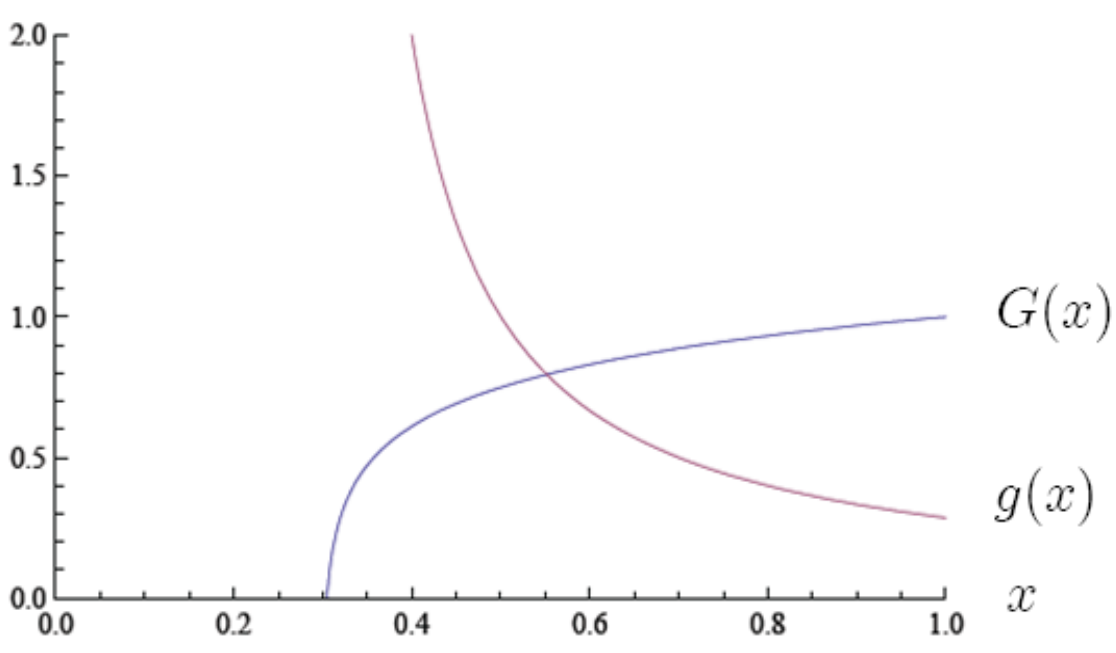

b) $\alpha=0.7$ and $\beta=0.3$

Figure 2. CDF (Blue) and PDF (Red) of $G$ for $\alpha>0.5>\beta$ when $\gamma=\delta=1$. 


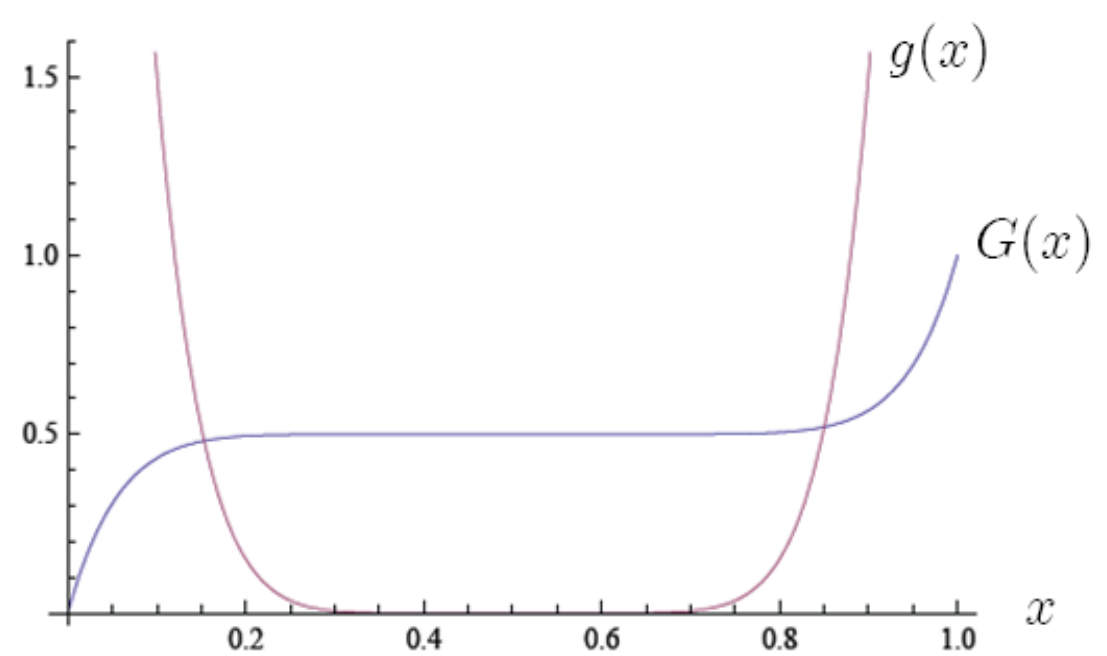

a) $\alpha=0.05$ and $\beta=0.05$

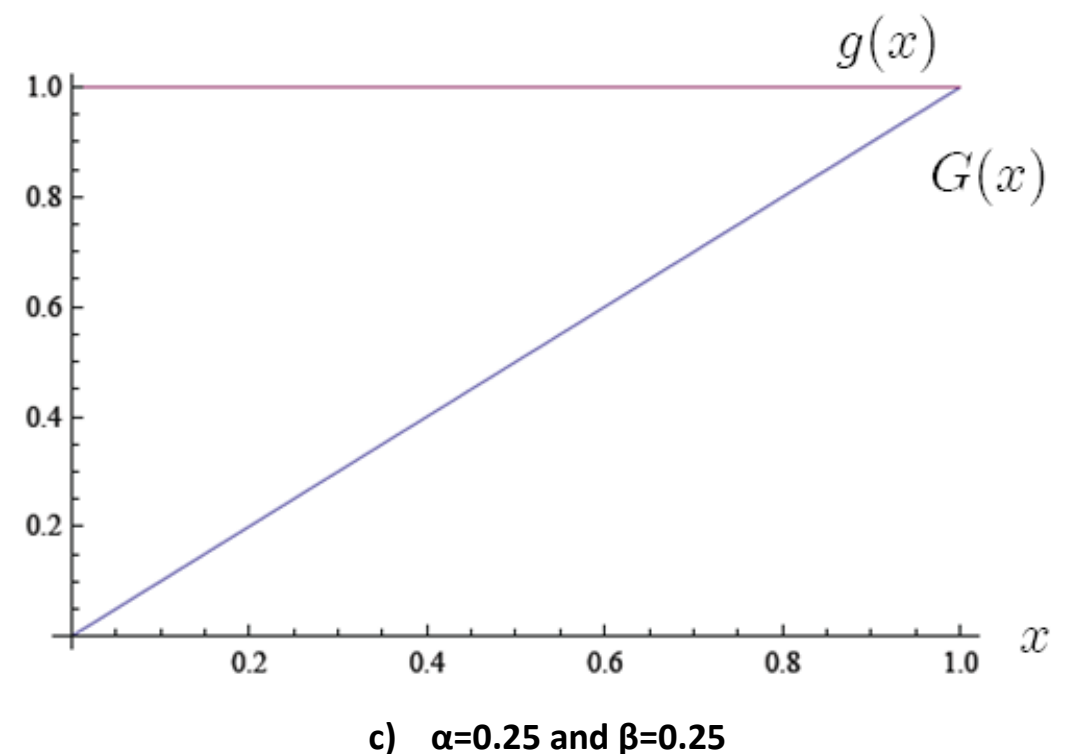

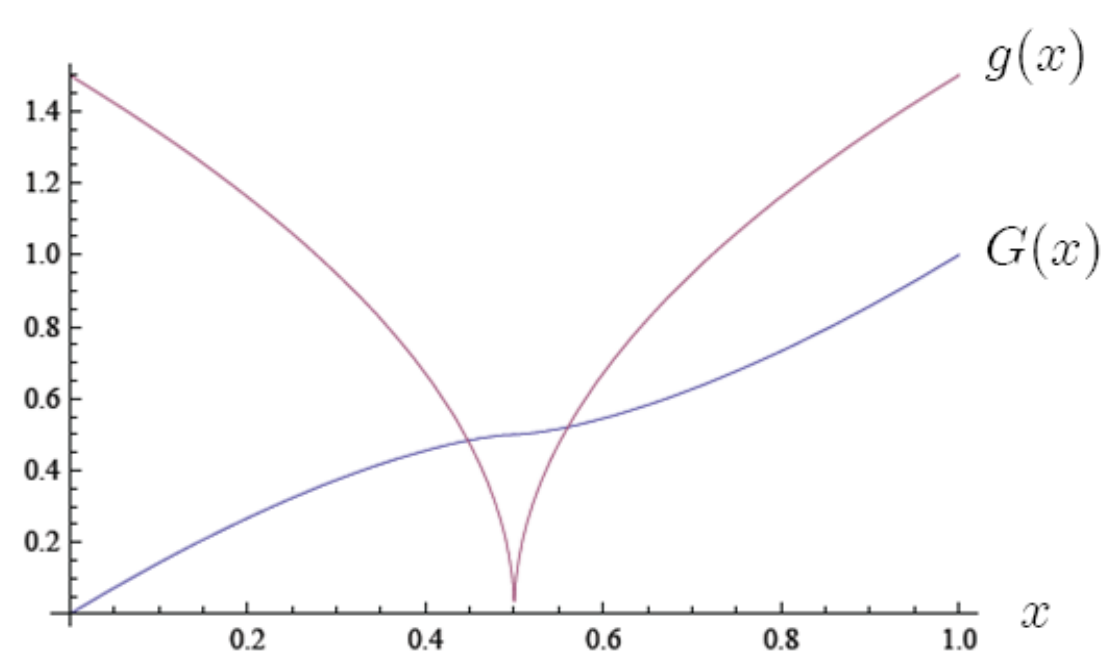

b) $\alpha=0.2$ and $\beta=0.2$

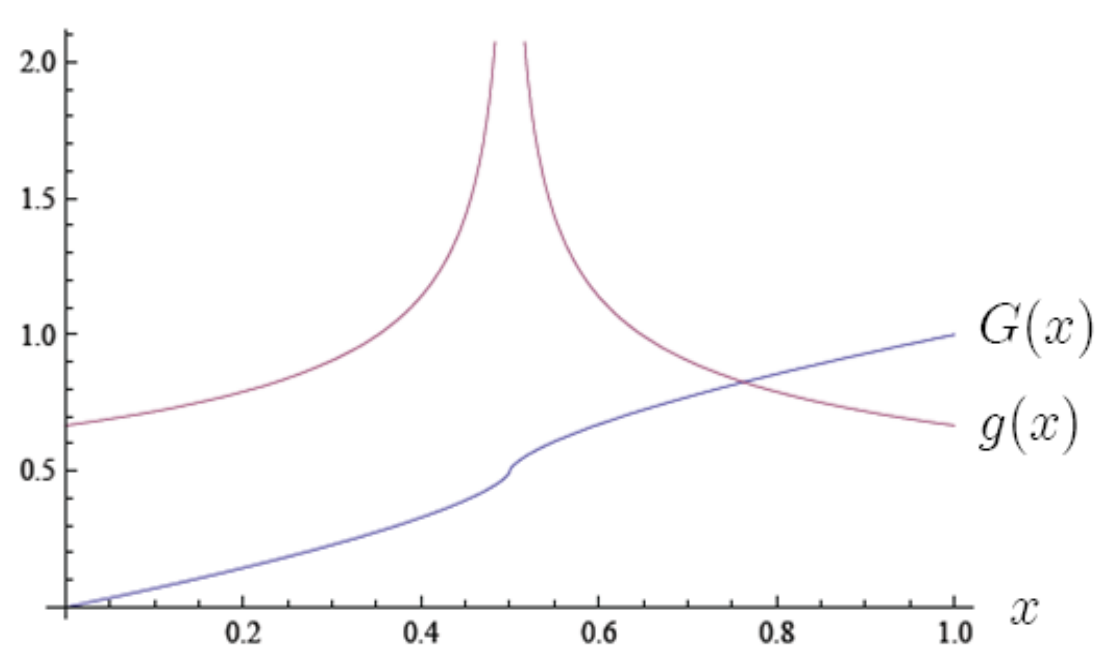

d) $\alpha=0.3$ and $\beta=0.3$

Figure 3. CDF (Blue) and PDF (Red) of $G$ for $\alpha, \beta<0.5$ when $\gamma=\delta=1$. 